\title{
SPATIAL EPIDEMICS: CRITICAL BEHAVIOR IN ONE DIMENSION
}

\author{
STEVEN P. LALLEY
}

\begin{abstract}
In the simple mean-field $S I S$ and $S I R$ epidemic models, infection is transmitted from infectious to susceptible members of a finite population by independent $p$-coin tosses. Spatial variants of these models are considered, in which finite populations of size $N$ are situated at the sites of a lattice and infectious contacts are limited to individuals at neighboring sites. Scaling laws for these models are given when the infection parameter $p$ is such that the epidemics are critical. It is shown that in all cases there is a critical threshold for the numbers initially infected: below the threshold, the epidemic evolves in essentially the same manner as its branching envelope, but at the threshold evolves like a branching process with a size-dependent drift. The corresponding scaling limits are super-Brownian motions and Dawson-Watanabe processes with killing, respectively.
\end{abstract}

\section{INTRODUCTION}

1.1. Critical mean-field epidemics: threshold behavior. It was discovered by Martin-Löf 18 and independently by Aldous [1] that the simple mean-field SIR epidemic, also known as the ReedFrost epidemic, exhibits a curious threshold behavior at criticality. Roughly, if $U_{N}$ is the size - that is, the number of individuals ever infected - of the epidemic in a population of size $N$, then $U_{N}$ has a markedly different asymptotic distribution when the number $J_{0}$ of individuals infected at time 0 is of order $N^{1 / 3}$ than when it is of order $o\left(N^{1 / 3}\right)$. In particular, if $J_{0} \sim b N^{\alpha}$ as $N \rightarrow \infty$, then

$$
U_{N} / N^{2 \alpha} \stackrel{\mathcal{D}}{\longrightarrow} \tau_{b},
$$

where $\tau_{b}$ is the first passage time to the level $b$ by a standard Wiener Process, if $\alpha<1 / 3$, or by a Wiener process with time-dependent drift $t$, if $\alpha=1 / 3$. This reflects the fact that the size of the largest connected component in a critical $(p=1 / N)$ Erdös-Renyi random graph on $N$ vertices is of order $N^{1 / 3}$. R. Dolgoarshinnykh and the author [9] have observed that there is a similar critical threshold effect for the simple mean-field $S I S$ epidemic, but here the threshold for $J_{0}$ is at $N^{1 / 2}$ rather than $N^{1 / 3}$, and the limit distribution at the threshold involves first passage times by Ornstein-Uhlenbeck processes. In fact, there is an asymptotic form for the entire evolution of the epidemic at criticality that undergoes a discontinuity at $J_{0} \approx N^{1 / 2}$ : If $J_{n}$ denotes the number of individuals infected at time $n$ then

$$
N^{-\alpha} J_{\left[N^{\alpha} t\right]} \stackrel{\mathcal{D}}{\longrightarrow} Y_{t}
$$

where $Y_{0}=b$ and $Y_{t}$ is either a Feller diffusion or a Feller diffusion with location-dependent drift $-Y_{t}^{2} d t$, depending on whether $\alpha<1 / 2$ or $\alpha=1 / 2$, that is,

$$
\begin{aligned}
& d Y_{t}=\sqrt{Y_{t}} d W_{t} \quad \text { if } \alpha<1 / 2 ; \\
& d Y_{t}=-Y_{t}^{2} d t+\sqrt{Y_{t}} d W_{t} \quad \text { if } \alpha=/ 2 .
\end{aligned}
$$

Date: October 10, 2018.

1991 Mathematics Subject Classification. Primary 60H30, secondary 60K35.

Key words and phrases. Spatial epidemic, branching random walk, Dawson-Watanabe process, critical scaling.

Supported by NSF grant DMS-0405102. 
Note that in the case $\alpha<1 / 2$ the limit process - the Feller diffusion - is the same as the limit process for the rescaled critical Galton-Watson process. There is a similar process-level threshold effect for the Reed-Frost epidemic at $J_{0} \approx N^{1 / 3}$ - see 9 .

1.2. Spatial $\boldsymbol{S I S}$ and $\boldsymbol{S I R}$ epidemics. The purpose of this article is to show that there is a similar critical threshold effect for spatial epidemics in one spatial dimension - see Theorem 1 below. The epidemic models considered are simple discrete-time spatial analogues of the Reed-Frost and stochastic logistic epidemics. These are chosen primarily to streamline the mathematical analysis; however, analogous effects should also be expected for more complex models in dimension $d=1$. A secondary motivation for the specification of the spatial SIR model is that it has a percolation (random graph) description similar to the Erdös-Renyi random graph description of the Reed-Frost epidemic, and so our main result can be interpreted as a statement about the connected clusters in certain percolation models.

The spatial epidemics, which we will henceforth call the $S I R-d$ and $S I S-d$ epidemics, are defined as follows: Assume that at each lattice point $x \in \mathbb{Z}^{d}$ is a homogeneous population ("village") of $N$ individuals, each of whom may at any time be either susceptible or infected, or (in the SIR variants) recovered. As in the corresponding mean-field models (see [18]), infected individuals remain infected for one unit of time, and then recover; in the SIR- $d$ epidemic, infected individuals recover and are thereafter immune from infection, while in the SIS-d model, infected individuals, upon recovery, become once again susceptible to infection. The rules of infection are the same as for the corresponding mean-field models, except that the infection rates depend on the locations of the infected and susceptible individuals. Thus, at each time $t=0,1,2, \ldots$, for each pair $\left(i_{x}, s_{y}\right)$ of an infected individual located at $x$ and a susceptible individual at $y$, the disease spreads from $i_{x}$ to $s_{y}$ with probability $p_{N}(x, y)$. We shall only consider the case where the transmission probabilities $p_{N}(x, y)$ are spatially homogeneous, nearest-neighbor, and symmetric, and scale with the village size $N$ in such a way that the expected number of infections by a contagious individual in an otherwise healthy population is 1 (so that the epidemic is critical), that is,

\section{Assumption 1.}

$$
\begin{aligned}
p_{N}\left(x, x+e_{i}\right) & =C_{d} / N \quad \text { if }\left|e_{i}\right|=1 \text { or } 0, \text { where } \\
C_{d} & =1 /(2 d+1) .
\end{aligned}
$$

Similar models incorporating separated clusters have been studied by Schinazi [21, Belhadji \& Lanchier [3], and others, but these studies have focused on SIS variants of the models where all infected individuals in a colony recover simultaneously, and where infection rates within and between colonies vary. Critical behavior of certain spatial epidemic models has been addressed in the literature, in particular for long-range contact processes [20, 12, which are in certain respects similar to the SIS- $d$ model described above; however, critical behavior of spatial SIR models has not been previously studied. For surveys of contact models in spatial epidemics, see [19] and [10].

Interest in spatial epidemic models has largely been focused on dimensions $d \geq 2$, and especially $d=2$, for natural reasons. Nevertheless, nearest neighbor infection models in dimension $d=1$ may be of interest in certain contexts: Many plant and animal species live in river valleys or along shorelines, and for these the natural dimension for spatial interactions is $d=1$.

1.3. Epidemic Models and Random Graphs. The models described above have equivalent descriptions as structured random graphs, that is, percolation processes. Consider first the simple SIR (Reed-Frost) epidemic. In this model, no individual may be infected more than once; furthermore, for any pair $x, y$ of individuals, there will be at most one opportunity for infection to pass from $x$ to $y$ or from $y$ to $x$ during the course of the epidemic. Thus, one could simulate the epidemic by first 
tossing a $p$-coin for every pair $x, y$, drawing an edge between $x$ and $y$ for each coin toss resulting in a Head, and then using the resulting (Erdös-Renyi) random graph determined by these edges to determine the course of infection in the epidemic. In detail: If $Y_{0}$ is the set of infected individuals at time 0 , then the set $Y_{1}$ of individuals infected at time 1 consists of all $x \notin Y_{0}$ that are connected to individuals in $Y_{0}$, and for any subsequent time $n$, the set $Y_{n+1}$ of individuals infected at time $n+1$ consists of all $x \notin \cup_{j \leq n} Y_{j}$ who are connected to individuals in $Y_{n}$. Note that the set of individuals ultimately infected during the course of the epidemic is the union of those connected components of the random graph containing at least one vertex in $Y_{0}$.

Similar random graph descriptions may be given for the mean-field $S I S$ and the spatial $S I S$ and $S I R$ epidemic models. Consider for definiteness the $S I R-d$ epidemic. To simulate this, first build a random graph by Bernoulli bond percolation on the graph $\mathbb{K}_{N} \times \mathbb{Z}^{d}$, where $\mathbb{K}_{N}$ is the complete graph on $N$ vertices. Given the random graph, simulate the generations $Y_{n}$ of the $S I R$ - $d$ epidemic by the same rule as in the mean-field case: For each generation $n$, define the set $Y_{n+1}$ of individuals infected at time $n+1$ to be the set of all vertices $x \notin \cup_{j}{ }_{n} Y_{j}$ who are connected to individuals in $Y_{n}$. Similar random graph descriptions may be given for $S I S$ epidemics, but using oriented percolation for the random graphs.

1.4. Branching envelopes of spatial epidemics. The branching envelope of a spatial SIS-d or $S I R-d$ epidemic is a branching random walk on the integer lattice $\mathbb{Z}^{d}$. This evolves as follows: Any particle located at site $x$ at time $t$ lives for one unit of time and then reproduces, placing random numbers $\xi_{y}$ of offspring at the sites $y$ such that $|y-x| \leq 1$. The random variables $\xi_{y}$ are mutually independent, each with Binomial- $\left(N, C_{d} / N\right)$ distributions, where $C_{d}=1 /(2 d+1)$. Denote this reproduction rule by $\mathcal{R}_{N}$, and denote by $\mathcal{R}_{\infty}$ the corresponding offspring law in which the Binomial distribution is replaced by the Poisson distribution with mean $C_{d}$. Note that for each of the offspring distributions $\mathcal{R}_{N}$, the branching random walk is critical, that is, the expected total number of offspring of a particle is 1 .

A fundamental theorem of S. Watanabe 24] asserts that, under suitable rescaling (the Feller scaling) the measure-valued processes naturally associated with critical branching random walks converge to a limit, the standard Dawson-Watanabe process, also known as super Brownian motion.

Definition 1. The Feller-Watanabe scaling operator $\mathcal{F}_{k}$ scales mass by $1 / k$ and space by $1 / \sqrt{k}$, that is, for any finite Borel measure $\mu(d x)$ on $\mathbb{R}^{d}$ and any test function $\phi(x)$,

$$
\left\langle\phi, \mathcal{F}_{k} \mu\right\rangle=k^{-1} \int \phi(\sqrt{k} x) \mu(d x)
$$

Watanabe's Theorem . Fix $N$, and for each $k=1,2, \ldots$ let $Y_{t}^{k}$ be a branching random walk with offspring distribution $\mathcal{R}_{N}$ and initial particle configuration $Y_{0}^{k}$. (In particular, $Y_{t}^{k}(x)$ denotes the number of particles at site $x \in \mathbb{Z}$ in generation $[t]$, and $Y_{t}^{k}$ is the corresponding Borel measure on $\mathbb{R}$.) If the initial mass distributions converge, after rescaling, as $k \rightarrow \infty$, that is, if

$$
\mathcal{F}_{k} Y_{0}^{k} \Longrightarrow X_{0}
$$

for some finite Borel measure $X_{0}$ on $\mathbb{R}^{d}$, then the rescaled measure-valued processes $\mathcal{F}_{k} Y_{k t}^{k}$ converge in law as $k \rightarrow \infty$ :

$$
\left(\mathcal{F}_{k} Y^{k}\right)_{k t} \Longrightarrow X_{t}
$$

The limit is the standard Dawson-Watanabe process $X_{t}$ (also known as super-Brownian motion). See 13 for more. In dimension $d=1$ the random measure $X_{t}$ is for each $t$ absolutely continuous relative to Lebesgue measure [17, and the Radon-Nikodym derivative $X(t, x)$ is jointly continuous in $t, x$ (for $t>0$ ). In dimensions $d \geq 2$ the measure $X_{t}$ is almost surely singular, and is supported by a Borel set of Hausdorff dimension 2 [7]. 
1.5. Scaling limits of critical spatial epidemics. Our main result, Theorem 1 below, asserts that after appropriate rescaling the $S I S-1$ and $S I R-1$ spatial epidemics converge weakly as $N \rightarrow \infty$. The limit processes are either standard Dawson-Watanabe processes or Dawson-Watanabe processes with variable-rate killing, depending on the initial configuration of infected individuals. The DawsonWatanabe process $X_{t}$ with killing rate $\theta=\theta(x, t, \omega)$ (assumed to be progressively measurable and jointly continuous in $t, x)$ and variance parameter $\sigma^{2}$ can be characterized by a martingale problem ([8], sec. 6.2): for every $\phi \in C_{c}^{2}\left(\mathbb{R}^{d}\right)$,

$$
\left\langle X_{t}, \phi\right\rangle-\left\langle X_{0}, \phi\right\rangle-\frac{\sigma_{d}}{2} \int_{0}^{t}\left\langle X_{s}, \Delta \phi\right\rangle d s+\int_{0}^{t}\left\langle X_{s}, \theta(\cdot, s) \phi\right\rangle d s
$$

is a martingale with the same quadratic variation as for the standard Dawson-Watanabe process. Existence and distributional uniqueness of such processes in general is asserted in [8] and proved, in various cases, in [6] and [14. It is also proved in these articles that the law of a DawsonWatanabe process with killing on a finite time interval is absolutely continuous with respect to that of a standard Dawson-Watanabe process with the same variance parameter, and that the likelihood ratio (Radon-Nikodym derivative) is 8

$$
\exp \left\{-\int \theta(t, x) d M(t, x)-\frac{1}{2} \int\left\langle X_{t}, \theta(t, \cdot)^{2}\right\rangle d t\right\},
$$

where $d M(t, x)$ is the orthogonal martingale measure attached to the standard Dawson-Watanabe process (see [23] and sec. 2.5] below). Absolute continuity implies that sample path properties are inherited: In particular, if $X_{t}$ is a one-dimensional Dawson-Watanabe process with killing, then almost surely the random measure $X_{t}$ is absolutely continuous, with density $X(x, t)$ jointly continuous in $x$ and $t$.

Theorem 1. Let $Y_{t}^{N}(x)$ be the number infected at time $t$ and site $x$ in a critical SIS-1 or SIR-1 epidemic with village size $N$ and initial configuration $Y_{0}^{N}(x)$. Fix $\alpha>0$, and let $X^{N}(t, x)$ be the renormalized particle density function process obtained by linear interpolation in $x$ from the values

$$
X^{N}(t, x):=\frac{Y_{\left[N^{\alpha} t\right]}^{N}\left(\sqrt{N^{\alpha}} x\right)}{\sqrt{N^{\alpha}}} \text { for } \quad x \in \mathbb{Z} / \sqrt{N^{\alpha}} .
$$

Assume that there is a compact interval $J$ such that the initial particle density functions $X^{N}(0, x)$ all have support contained in $J$, and assume that the functions $X^{N}(0, x)$ converge in $C_{b}(\mathbb{R})$ to a function $X(0, x)$. Then under Assumption 1 , as $N \rightarrow \infty$,

$$
X^{N}(t, x) \Longrightarrow X(t, x)
$$

where $X(t, x)$ is the density of a Dawson-Watanabe process $X_{t}$ with initial density $X(0, x)$ and killing rate $\theta$ depending on the value of $\alpha$ and the type of epidemic (SIS or SIR) as follows:

(a) SIS: If $\alpha<\frac{2}{3}$ then $\theta(x, t)=0$.

(b) SIS: If $\alpha=\frac{2}{3}$ then

$$
\theta(x, t)=X(x, t) / 2 .
$$

(c) SIR: If $\alpha<\frac{2}{5}$ then then $\theta(x, t)=0$.

(d) SIR: If $\alpha=\frac{2}{5}$ then

$$
\theta(x, t)=\int_{0}^{t} X(x, s) d s .
$$

The convergence $\Rightarrow$ in (12) is weak convergence relative to the Skorohod topology on the space $\mathbb{D}\left([\cup, \infty), C_{b}(\mathbb{R})\right)$ of cadlag functions $t \mapsto X(t, x)$ valued in $C_{b}(\mathbb{R})$. 
The proof of Theorem 1 is given in section 2, it will will depend on Theorem 2 below. In both Theorems 2 and 1, the assumption that the initial particle densities have common compact support $J$ can undoubtedly be weakened; however, this assumption eliminates certain technical complications in the arguments (see equation (27) in sec. 2).

Remarks. (A) The case of principal interest is the SIR-1 epidemic. The $S I S-1$ epidemic is closely related to the long-range contact process studied by Mueller and Tribe [20 in $d=1$ and by Durrett and Perkins [12] in $d \geq 2$, and in particular the limit process for the SIS-1 epidemic, the DawsonWatanabe process with killing rate (13), is the same as that for the rescaled contact process in $d=1$. Nevertheless, the rescaled contact process and the discrete-time SIS-1 epidemic studied here differ in some important technical respects, so parts (a)- (b) of Theorem 1 do not follow from the results of [20].

(B) The proof strategy here (sec. 2 below) is considerably different - and simpler - than the usual approach, based on martingale methods, taken in the literature of weak convergence to superprocesses, such as in [20, 12, [5, and [11. Although martingale methods might be made to work here, the prospect of using them in connection with processes such as the SIR-1 epidemic with history-dependent rates is rather daunting. Instead, we rely on the fact that the laws of both the SIS and SIR epidemics are absolutely continuous with respect to those of their branching envelopes, for which scaling limits are already known. The Radon-Nikodym derivatives have tractable forms, as exponentials of certain stochastic integrals. These will be shown to converge to the corresponding Radon-Nikodym derivatives (10). The advantage of this strategy is that, given Theorem 2, there is no need to check tightness for the rescaled epidemic processes. Moreover, there is virtually no additional work involved in establishing the result for the SIS model - all that is needed is an additional simple asymptotic estimation of the Radon-Nikodym derivatives.

(C) In higher dimensions, there is no analogous threshold effect at criticality. In dimensions $d \geq 3$, a branching random walk started by any number of particles will quickly diffuse, so that after a short time most occupied sites will have only $O(1)$ particles. Consequently, in both the SIS- $d$ and the $S I R-d$ epidemics, the effect of finite population size on the production of new infections will be limited, and so both epidemics will behave in more or less the same manner as their branching envelopes. In dimension $d=2$, the situation is somewhat more interesting: it appears that here finite population size will manifest itself by a logarithmic drag on the production of new particles. The cases $d \geq 2$ will be discussed in detail in a forthcoming paper of Xinghua Zheng [26].

1.6. Heuristics: The standard coupling. The critical thresholds for the $S I S-d$ and $S I R-d$ epidemics can be guessed by a simple comparison argument based on the standard coupling of the epidemic and its branching envelope. For the $S I S-d$ epidemic, the coupling is constructed as follows: Build a branching random walk whose initial state coincides with that of the epidemic, with particles to be colored red or blue according to whether or not they represent infections that actually take place (red particles represent actual infections). Initially, all particles are red. At each time $t=0,1,2, \ldots$, particles of the branching random walk produce offspring at neighboring sites according to the law described in sec. 1.4 above. Offspring of blue particles are always blue, but offspring of red particles may be either red or blue, with the choices made as follows: All offspring of red particles at a location $y$ choose labels $j \in[N]$ at random, independently of all other particles; for any label $j$ chosen by $k>1$ particles, one of the particles is chosen at random and colored red, and the remaining $k-1$ particles are colored blue. The population of all particles evolves as a branching random walk, by construction, while the subpopulation of red particles evolves as an $S I S-d$ epidemic. Observe that the branching random walk dominates the epidemic: thus, the duration, size, and spatial extent of the epidemic are limited by those of the branching envelope. 
The standard coupling of an $S I R-d$ epidemic with its branching envelope is constructed in a similar fashion, but with the following rule governing choices of color by offspring of red particles: All offspring of red particles at a location $y$ choose numbers $j \in[N]$ at random, independently of all other particles. If a particle chooses a number $j$ that was previously chosen by a particle of an earlier generation at the same site $y$, then it is assigned color blue. If $k>1$ offspring of red particles choose the same number $j$ at the same time, and if $j$ was not chosen in an earlier generation, then 1 of the particles is assigned color red, while the remaining $k-1$ are assigned color blue. Under this rule, the subpopulation of red particles evolves as an $S I R-d$ epidemic.

In both couplings, the production of blue offspring by red particles may be viewed as an attrition of the red population. Assume that initially there are $N^{\alpha}$ particles; then by Feller's limit theorem for critical Galton-Watson processes, the branching envelope can be expected to survive for $O_{P}\left(N^{\alpha}\right)$ generations, and at any time prior to extinction the population will have $O_{P}\left(N^{\alpha}\right)$ members. These will be distributed among the sites at distance $O_{P}\left(N^{\alpha / 2}\right)$ from the origin, and therefore in dimension $d=1$ there should be about $O_{P}\left(N^{\alpha / 2}\right)$ particles per site. Consequently, for the $S I S-1$ epidemic, the rate of attrition per site per generation should be $O_{P}\left(N^{\alpha-1}\right)$, and so the total attrition rate per generation should be $O_{P}\left(N^{3 \alpha / 2-1}\right)$. If $\alpha=2 / 3$, then the total attrition rate per generation will be $O_{P}(1)$, just enough so that the total attrition through the duration of the branching random walk envelope will be on the same order of magnitude as the population size $N^{\alpha}$.

For the $S I R-1$ epidemic there is a similar heuristic calculation. As for the $S I S-1$ epidemic, the branching envelope will survive for $O_{P}\left(N^{\alpha}\right)$ generations, and up to the time of extinction the population should have $O_{P}\left(N^{\alpha}\right)$ individuals, about $O_{P}\left(N^{\alpha / 2}\right)$ per site. Therefore, through $N^{\alpha}$ generations, about $N^{\alpha} \times N^{\alpha / 2}$ numbers $j$ will be retired, and so the attrition rate per site per generation should be $O_{P}\left(N^{\alpha / 2} \times N^{3 \alpha / 2}\right)$, making the total attrition rate per generation $O_{P}\left(N^{5 \alpha / 2}\right)$. Hence, if $\alpha=2 / 5$ then the total attrition per generation should be $O_{P}(1)$, just enough so that the total attrition through the duration of the branching random walk envelope will be on the same order of magnitude as the population size.

1.7. Weak convergence in $\mathbb{D}\left([0, \infty), C_{b}(\mathbb{R})\right)$. The heuristic argument above has an obvious gap: it relies crucially on the assertion that the particles of a critical branching random walk distribute themselves somewhat uniformly, at least locally, among the sites at distance $N^{\alpha / 2}$ from the origin. The fact that the Dawson-Watanabe process in dimension one has a continuous density suggests that this should be true, but does not imply it. Following is a strengthening of the Watanabe theorem suitable for our purposes.

Denote by $C_{b}(\mathbb{R})$ the space of continuous, bounded, real-valued functions on $\mathbb{R}$ with the sup-norm topology, and by $\mathbb{D}\left([0, \infty), C_{b}(\mathbb{R})\right)$ the Skorohod space of cadlag functions $X(t, x)$ valued in $C_{b}(\mathbb{R})$ (thus, for each $t \geq 0$ the function $X(t, x)$ is a continuous, bounded function of $x$ ). Fix $N \leq \infty$, and for $k=1,2, \ldots$ let $Y_{t}^{k}(x)$ be the number of particles at site $x$ at time $[t]$ in a branching random walk with offspring distribution $\mathcal{R}_{N}$ and initial particle configuration $Y_{0}^{k}(\cdot)$. Let $X^{k}(t, x)$ be the renormalized density function: that is, the function obtained by linear interpolation (in $x$ ) from the values

$$
X^{k}(t, x)=\frac{Y_{[k t]}^{k}(\sqrt{k} x)}{\sqrt{k}} \text { for } \quad x \in \mathbb{Z} / \sqrt{k} .
$$

Theorem 2. Assume that there is a compact interval $J \subset \mathbb{R}$ such that all of the the initial particle densities $X^{k}(0, \cdot)$ have support $\subset J$, and assume that as $k \rightarrow \infty$ the functions $X^{k}(0, \cdot)$ converge in $C_{b}(\mathbb{R})$ to a continuous function $X(0, \cdot)$. Then as $k \rightarrow \infty$,

$$
X^{k}(t, x) \Longrightarrow X(t, x),
$$


where $X(t, x)$ is the density function of a Dawson-Watanabe process with initial density $X(0, x)$, and $\Rightarrow$ indicates weak convergence relative to the Skorohod topology on $\mathbb{D}\left([0, \infty), C_{b}(\mathbb{R})\right)$.

To prove this, it suffices to show that the sequence $X^{k}(t, x)$ of densities is tight in $\mathbb{D}\left([0, \infty), C_{b}(\mathbb{R})\right)$, because Watanabe's theorem implies that any weak limit of a subsequence must be a density of the Dawson-Watanabe process. The proof of tightness, carried out in section 3 below, will be based on a form of the Kolmogorov-Chentsov tightness criterion and a moment calculation. This proof will use only three properties of the offspring law $\mathcal{R}_{N}$ :

(a) the mean number of offspring is 1 ;

(b) the total number of offspring has finite $m$ th moment, for each $m \geq 1$; and

(c) offspring choose locations at random from among the neighboring sites.

Since the $m$ th moments are bounded uniformly over the class of Binomial- $(N, 1 / N)$ distributions, it will follow that tightness holds simultaneously for all of the offspring laws $\mathcal{R}_{N}$. Only the case $N=\infty$ will be needed for the analysis of the spatial epidemic models, however.

Remark. Mueller and Tribe 20] proved that the density processes of rescaled long-range contact processes in one dimension converge weakly to the density process of a Dawson-Watanabe process with killing, but make no explicit use of branching random walks in their argument. Nevertheless, they likely were aware that the density processes of rescaled branching random walks would also converge weakly in one dimension.

1.8. Spatial extent of the Dawson-Watanabe process. An object of natural interest in connection with the spatial SIS and SIR epidemics is the spatial extent of the process, that is, the area reached by the infection. Under certain natural restrictions on the initial configuration of infected individuals, the spatial extent will, by Theorem 1 be well-approximated in law by the area covered by the limiting Dawson-Watanabe process, after suitable scaling. For Dawson-Watanabe processes $X_{t}$ with location-dependent killing, the distribution of $\mathcal{R}(X):=\cup_{t} \operatorname{support}\left(X_{t}\right)$ likely cannot be described in closed form. However, for the Dawson-Watanabe process with constant killing rate, the distribution of $\mathcal{R}(X)$ can be given in a computable form, as we now show.

Proposition 1. Let $X_{t}$ be the standard one-dimensional Dawson-Watanabe process with variance parameter $\sigma^{2}=1$. For any finite Borel measure $\mu$ with support contained in the interval $D=(0, a)$,

$$
-\log P\left(\mathcal{R}(X) \subset D \mid X_{0}=\mu\right)=\int \wp_{L}(x / \sqrt{6}) \mu(d x)
$$

where

$$
\wp_{L}(x)=\sum_{\omega \in L \backslash\{0\}}\left\{\frac{1}{6(x-\omega)^{2}}-\frac{1}{\omega^{2}}\right\}
$$

is the Weierstrass $\wp$-function with period lattice $L$ generated by $\sqrt{6} a e^{\pi i / 3}$.

Proof. By a theorem of Dynkin [13], ch. 8, the function

$$
u_{D}(x):=-\log P\left(\mathcal{R}(X) \subset D \mid X_{0}=\delta_{x}\right)
$$

is the unique solution of the differential equation $u^{\prime \prime}=u^{2}$ in $D$ with boundary conditions $u(x) \rightarrow \infty$ as $x \rightarrow 0, a$. Set $v=u^{\prime}$; then the equation $u^{\prime \prime}=u^{2}$ becomes $v^{\prime}=u^{2}$, and so $d v / d u=u^{2} / v$. Integration gives

$$
v^{2} / 2=u^{3} / 3+C,
$$

which, up to constants, is the differential equation of the $\wp$-function [25], ch. 20. The result (17) now follows for the special case $\mu=\delta_{x}$. The general case now follows by the superposition principle for branching processes: in particular, the Dawson-Watanabe process $X_{t}$ with initial condition 
$X_{0}=\mu+\nu$ can be decomposed as the union of independent Dawson-Watanabe processes with initial conditions $\mu, \nu$, respectively, and so the quantity on the left side of (17) is linear in $\mu$. It follows that (17) holds for all initial measures $\mu$ with support contained in $D$.

\section{Spatial Epidemic Models: Proof of Theorem 1}

2.1. Strategy. We shall exploit the fact that the laws of the spatial $S I S$ and $S I R$ epidemics are absolutely continuous with respect to the laws of their branching envelopes. Because the branching envelopes converge weakly, after rescaling, to super-Brownian motions, by Watanabe's theorem (and in the stronger sense of Theorem 22), to prove the weak convergence of the rescaled spatial epidemics it will suffice to show that the likelihood ratios converge, in a suitable sense, to the likelihood ratios of the appropriate Dawson-Watanabe processes relative to super-Brownian motion:

Proposition 2. Let $X_{n}, X$ be random variables valued in a metric space $\mathcal{X}$, all defined on a common probability space $(\Omega, \mathcal{F}, P)$, and let $L_{n}, L$ be nonnegative, real-valued random variables on $(\Omega, \mathcal{F}, P)$ such that

$$
E_{P} L_{n}=E_{P} L=1 \quad \forall n
$$

Let $Q_{n}, Q$ be the probability measures on $(\Omega, \mathcal{F})$ with likelihood ratios $L_{n}, L$ relative to $P$. If

$$
\left(X_{n}, L_{n}\right) \Longrightarrow(X, L)
$$

under $P$ as $n \rightarrow \infty$, then the $Q_{n}$-distribution of $X_{n}$ converges to the $Q$-distribution of $X$, that is, for every bounded continuous function $f: \mathcal{X} \rightarrow \mathbb{R}$,

$$
\lim _{n \rightarrow \infty} E_{Q_{n}} f\left(X_{n}\right)=E_{Q} f(X)
$$

Proof. Routine.

Recall [6 that the law of the Dawson-Watanabe process with killing is absolutely continuous with respect to that of the standard Dawson-Watanabe process, with likelihood ratio given by (10). The likelihood ratio involves stochastic integration with respect to an orthogonal martingale measure; thus, the obvious strategy for proving (21) in our context is to express the likelihood ratios of the spatial epidemic processes in terms of stochastic integrals against the OMMs of the branching envelopes. Although it is possible to work directly with the likelihood ratios of the epidemic processes to their branching envelopes, this is somewhat messy, for two reasons: (A) the offspring distributions $\mathcal{R}_{N}$ of the branching envelopes change with the village size $N$; and (B) the random mechanism by which particles of the branching envelope are culled in the standard coupling involves dependent Bernoulli random variables. Therefore, we will first show, by comparison arguments, that the spatial epidemic processes can be modified so that difficulties (A) and (B) are circumvented, and in such a way that asymptotic behavior is not affected. The likelihood ratios of the modified processes relative to critical Poisson branching random walks will then be computed in sec. 2.6.

2.2. Extent, duration, size, and density of the branching envelope. Since the spatial SIS and SIR epidemics are stochastically dominated by their branching envelopes, their durations, sizes, etc., are limited by those of their envelopes. For critical branching random walks, the scaling limit theorems of Watanabe and Feller give precise information about these quantities. Consider first the duration: Since the total mass in a $B R W$ is just a Galton-Watson process, if the branching random walk is initiated by $N^{\alpha}$ particles, then by a standard result in the theory of Galton-Watson processes (Th. I.9.1 of [2]), the time $T_{N}$ to extinction scales like $N^{\alpha}$, that is,

$$
T_{N} / N^{\alpha} \Longrightarrow F \text {. }
$$


The limit distribution $F$ is the distribution of the first passage time to 0 by a Feller diffusion process started at 1. Consequently, under the hypotheses of Theorem[1, the duration of the $B R W$ is $O_{P}\left(N^{\alpha}\right)$. Furthermore, if $Z_{n}^{N}$ is the mass in the $n$th generation (that is, the total number of particles), then by Feller's theorem ([13, ch. 1),

$$
Z_{\left[N^{\alpha} t\right]}^{N} / N^{\alpha} \Longrightarrow Z_{t}
$$

where $Z_{t}$ is a Feller diffusion process started at 1 . Consequently, the total mass produced during the entire course of the branching envelope is of order $O_{P}\left(N^{2 \alpha}\right)$ : in particular,

$$
\sum_{n} Z_{n}^{N} / N^{2 \alpha} \Longrightarrow \int_{0}^{\infty} Z_{t} d t
$$

Since the Feller diffusion is absorbed at 0 in finite time almost surely, the integral is finite with probability 1 .

Next, consider the maximal density and spatial extent of the branching random walk. Watanabe's theorem implies that if initially all particles are located in an interval of size $N^{\alpha / 2}$ centered at 0 , as required by Theorem 1, then the bulk of the mass must remain within $O_{P}\left(N^{\alpha / 2}\right)$ of the origin, because the limiting Dawson-Watanabe process has bounded support. A theorem of Kesten [16 implies that in fact all of the mass remains within $O_{P}\left(N^{\alpha / 2}\right)$ of the origin: that is, under the hypotheses of Theorem 1, if

$$
Y_{N}^{*}:=\max \left\{|x|: \sum_{t<\infty} Y_{t}^{N}(x)>0\right\},
$$

then for any $\varepsilon>0$ there exists $\beta<\infty$ such that

$$
P\left\{Y_{N}^{*} \geq \beta N^{\alpha / 2}\right\}<\varepsilon
$$

Together, (23) and (26) imply that if initially the branching random walk has $O\left(N^{\alpha}\right)$ particles all located at sites within distance $O\left(N^{\alpha / 2}\right)$ of the origin, then the number of site/time pairs $(x, t)$ reached by the branching random walk is $O_{P}\left(N^{3 \alpha / 2}\right)$. Now suppose in addition that the initial configurations satisfy the more stringent requirement $X^{N}(0, x) \Rightarrow X(0, x)$ of Theorem 1 then Theorem 2 implies that the renormalized density processes $X^{N}(t, x) \Rightarrow X(t, x)$, where $X(t, x)$ is the renormalized density process of the standard Dawson-Watanabe process. Since $X(t, x)$ is jointly continuous and has compact support [17, it follows that

$$
\max _{t, x} Y^{N}(t, x)=O_{P}\left(N^{\alpha / 2}\right) .
$$

2.3. Binomial/Poisson and Poisson/Poisson comparisons. In this section we show that, in the asymptotic regimes considered in Theorem 1 the $\operatorname{Binomial}-(N, 1 / N)$ random variables used in the the standard coupling (sec. (1.6) can be replaced by Poisson- 1 random variables without changing the asymptotic behavior of the density processes $X^{N}(t, x)$. Recall that in the standard coupling, each particle, whether red or blue, produces a random number of offspring with the Binomial- $(N, 1 / N)$ distribution. The total number of particles produced during the lifetime of the branching envelope is, under the hypotheses of Theorem 11, at most $O_{P}\left(N^{2 \alpha}\right)$, and $\alpha \leq 2 / 3$ in all scenarios considered, by (24). Consequently, if all of the Binomial- $1 / N$ random variables used in the construction were replaced by Poisson-1 random variables, the resulting processes (both the red process, representing the spatial epidemic, and the red+blue process, representing the branching envelope) would be indistinguishable from the original processes, by the following lemma. 
Lemma 1. Assume that under the probability measure $\mu_{N}$, the random variables $X_{1}, X_{2}, \ldots$ are i.i.d. Binomial- $(N, 1 / N)$, and that under measure $\nu$ they are i.i.d. Poisson-1. Let $m=m_{N}$ be a sequence of positive integers such that for some $\varepsilon>0$,

$$
m_{N}=O\left(N^{2-\varepsilon}\right)
$$

If $\mathcal{G}_{m}$ is the $\sigma$-algebra generated by $X_{1}, X_{2}, \ldots, X_{m}$, then

$$
\left(\frac{d \mu_{N}}{d \nu}\right)_{\mathcal{G}_{m}} \stackrel{\nu}{\longrightarrow} 1 \text {. }
$$

Proof. This is a routine calculation. Fix a sequence $x_{1}, x_{2}, \ldots, x_{m}$ of nonnegative integers; the likelihood ratio $d \mu_{N} / d \nu$ of this sequence is

$$
\prod_{i=1}^{m} \frac{N !(N-1)^{-x_{i}}}{\left(N-x_{i}\right) !}\left(1-N^{-1}\right)^{N} e^{1} .
$$

For $m=O\left(N^{2-\varepsilon}\right)$,

$$
\left\{\left(1-N^{-1}\right)^{N} e^{1}\right\}^{m} \sim \exp \{-m / 2 N\} .
$$

By Chebyshev's inequality and elementary calculus, for $\nu$-typical sequences $x_{i}$,

$$
\prod_{i=1}^{m} \frac{N !(N-1)^{-x_{i}}}{\left(N-x_{i}\right) !}=\exp \left\{\left(m+O_{P}(\sqrt{m})\right) / 2 N\right\} .
$$

Recall that in the standard coupling (sec. [1.6), red particles represent infections that occur in the spatial epidemic, whereas blue particles represent attempted infections that are suppressed because either two or more infected individuals try to infect the same susceptible simultaneously, or (in the $S I R$ case) because the target of the attempted infection has acquired immunity by dint of an earlier infection. It is possible that more than one attempted infection is suppressed at once, that is, more than one blue particle with a red parent is created at a given site/time. In sec. 2.4 below, we will show that such occurrences are sufficiently rare that their effect on the epidemic process is negligible in the large- $N$ limit. To do so, we will bound the set of offspring of such blue particles by the set of discrepancies between a Poisson branching random walk with mean offspring number $1+\varepsilon$ and one with mean 1 , for some small $\varepsilon$. The next result shows that if $\varepsilon$ is sufficiently small relative to the size (total number of particles) of the Poisson branching random walk, then the effect of changing the mean offspring number is negligible.

Lemma 2. Let $\mu_{K}$ and $\nu_{K}$ be the distributions of Poisson branching random walks with mean offspring numbers $1+\varepsilon_{K}$ and 1 , respectively, and common initial configuration $Y_{0}^{K}(x)$ with $K$ particles. If

$$
\varepsilon_{K}=o(1 / K)
$$

then under $\nu_{K}$, as $K \rightarrow \infty$.

$$
\frac{d \mu_{K}}{d \nu_{K}} \Longrightarrow 1
$$

Proof. For a given sample evolution in which $Z_{K}$ particles are created, the likelihood ratio of $\mu_{K}$ relative to $\nu_{K}$ is

$$
\frac{d \mu_{K}}{d \nu_{K}}=\left(1+\varepsilon_{K}\right)^{Z_{K}} \exp \left\{-\varepsilon_{K} Z_{K}\right\}=1+O\left(Z_{K} \varepsilon_{K}^{2}\right)
$$

Under $\nu_{K}$, the branching random walk will last on the order of $K$ generations, during which on the order of $K$ particles will be created in each generation, by Watanabe's theorem. Hence, $Z_{K}$ will 
typically be of size $K^{2}$. In fact, if $\xi_{n}^{K}$ is the number of particle creations in the $n$th generation, then $\xi_{n}^{K}$ is, under $\nu_{K}$, a Galton-Watson process with offspring distribution Poisson-1 and initial condition $\xi_{0}^{K}=K$, so Feller's theorem implies

$$
Z_{K} / K^{2} \Longrightarrow \int_{0}^{\infty} \xi_{t} d t
$$

where $\xi_{t}$ is a Feller diffusion process with initial state $\xi_{0}=1$. The assertion now follows from the hypothesis (31).

The measures $\mu_{K}, \nu_{K}$ can be coupled as follows: Start with an initial configuration $Y_{0}^{K}(x)$, as in the lemma, and let particles reproduce and move as in a branching random walk with offspring distribution Poisson- $\left(1+\varepsilon_{K}\right)$. Attach to each particle $\zeta$ a Bernoulli- $\varepsilon_{K} /\left(1+\varepsilon_{K}\right)$ random variable $U_{\zeta}$. Assign colors green or orange to particles according to the following rules: (A) Offspring of green particles are always green. (B) An offspring $\zeta$ of an orange particle is green if $U_{\zeta}=1$, otherwise is orange. Then the process of orange particles evolves as a branching random walk with offspring distribution Poisson-1, and the process of all particles, green and orange, evolves as a $B R W$ with offspring distribution Poisson- $\left(1+\varepsilon_{K}\right)$. Denote by $Y_{t}^{K, G}(x)$ the number of green particles at site $x$, time $t$, and by $X^{K, G}(t, x)$ the renormalized density function obtained from $Y_{t}^{K, G}(x)$ by the rule (15), with $k=K$.

Corollary 1. Assume that the initial configurations $Y_{0}^{K}(\cdot)$ of the branching random walks satisfy the hypotheses of Theorem Q If $\varepsilon_{K}=o(1 / K)$, then as $k \rightarrow \infty$,

$$
\max _{t, x} X^{K, G}(t, x) \Longrightarrow 0 \text {. }
$$

Proof. Denote by $X^{K}(t, x)$ the renormalized density process associated with the branching random walk of orange particles. By Theorem 2, the processes $X^{K} \Rightarrow X$ where $X=X(t, x)$ is the density of a standard Dawson-Watanabe process. By Lemma 2 the density $X^{K}+X^{K, G}$ of the orange+green particle branching random walk also converges to the density of a standard Dawson-Watanabe process. Since there is only one Dawson-Watanabe density process, it must be that $X^{K, G}$ converges weakly to zero in sup norm.

2.4. Multiple collisions. In the standard coupling (sec. 1.6) of a spatial SIS or SIR epidemic with its branching envelope, offspring of red particles at each site choose labels $j \in[N]$ at random, which are then used to determine colors as follows: (A) If an index $j$ is chosen by more than one particle, then all but one of these are colored blue. (B) (SIR model only) If index $j$ was chosen at the same site in an earlier generation, then all particles that choose $j$ are colored blue. We call events (A) or (B), where offspring of red particles are colored blue, collisions. At a site/time where there are $\geq 2$ blue offspring of red particles we say that a multiple collision has occurred. In this section, we show that for either SIS or SIR epidemics, up to the critical thresholds (see the statement of Theorem 1), the effects of multiple collisions on the evolution of the red particle-process are asymptotically negligible. In particular, this will justify replacing the standard coupling of sec. 1.6 by the following modification, in which at each time/site there is at most one blue offspring of a red parent.

Modified Standard Coupling: Particles are colored red or blue. Each particle produces a random number of offspring, according to the Poisson-1 distribution, which then randomly move either $+1,-1$, or 0 steps from their birth site. Once situated, these offspring are assigned colors according to the following rules:

(A) Offspring of blue particles are blue; offspring of red particles may be either red or blue.

(B) At any site/time $(x, t)$ there is at most one blue offspring of a red parent. 
(C) Given that there are $y$ offspring of red parents at site $x$, time $t$, the conditional probability $\kappa_{N}(y)=\kappa_{N, t, x}(y)$ that one of them is blue is

$$
\begin{aligned}
\kappa_{N}(y) & =y(y-1) /(2 N) \text { for } S I S \text { epidemics, and } \\
\kappa_{N}(y) & =y R / N \quad \text { for } S I R \text { epidemics, where } \\
R & =R_{t}^{N}(x)=\sum_{s<t} Y_{s}^{N}(x) .
\end{aligned}
$$

Here $Y_{t}^{N}(x)$ is the number of red particles at site $x$ in generation $t$, and so $R=R_{t}^{N}(x)$ is the number of recovered individuals at site $x$ at time $t$, equivalently, the number of labels $j \in[N]$ that have been used in the standard coupling at $x$ by time $t$. Observe that in both the SIS and the SIR cases, the value of $\kappa(y)$ is almost, but not exactly, equal to the conditional probability that in the standard coupling there would be at least one blue offspring of a red particle. The small discrepancies will make the expressions in the likelihood ratios (51) simpler.

The next result will justify replacing the standard coupling of sec. 1.6 by the modified standard coupling.

Proposition 3. The standard couplings and the modified standard couplings can be constructed simultaneously in such a way that the following is true, for initial configurations satisfying the hypotheses of Theorem 1 . If $Y_{t}^{N, \Delta}(x)$ is the discrepancy between the numbers of red particles at $(x, t)$ in the standard and modified standard couplings, then under the hypotheses of Theorem 1 , as $N \rightarrow \infty$,

$$
\max _{t, x} Y^{N, \Delta}(t, x)=o_{P}\left(N^{\alpha / 2}\right) .
$$

Lemma 3. Let $B_{N}$ (B for "bad") be the number of sites/times $(x, t)$ where there are at least 4 blue offspring of red particles in the standard coupling. Then as $N \rightarrow \infty$,

$$
B_{N}=o_{P}(1) \text {. }
$$

Proof. Theorem 2 implies that, under the hypotheses of Theorem 1 the maximum number of particles (of any color) at any site/time in the standard coupling is $O_{P}\left(N^{\alpha / 2}\right)$. (Note: This also relies on the fact that the limiting Dawson-Watanabe density process $X(t, x)$ is continuous and has compact support, w.p.1.) Hence, we may restrict attention to sample evolutions where $y=y_{t}(x)$, the total number of offspring of red particles at $(x, t)$, satisfies $y \leq C N^{\alpha / 2}$ for some fixed constant $C<\infty$. Furthermore, by the considerations of sec. 2.2. we may restrict attention to sample evolutions of duration $\leq C N^{\alpha}$ and spatial extent $C N^{\alpha / 2}$. Since $\alpha \leq 2 / 3$, it follows that the number of pairs $(x, t)$ visited by particles of the branching envelope is no more than $C^{2} N$.

In order that there be at least 4 blue offspring of red parents at site $x$, time $t$, at least 4 pairs (possibly overlapping) of red-parent offspring must choose common labels $j \in[N]$. The conditional probability of this happening, given the value of $y=y_{t}(x)$, is no more than $C^{\prime} y^{8} / N^{4}$, for some constant $C^{\prime}$ not depending on $y$ or $N$. But $y \leq C N^{\alpha / 2}$, so this conditional probability is bounded by $C^{\prime \prime} N^{4 \alpha-4} \leq C^{\prime \prime} N^{-4 / 3}$. Since there are only $C^{2} N$ sites to consider, it follows that, on the event delineated in the preceding paragraph, the probability that $B_{N} \geq 1$ is $O\left(N^{-1 / 3}\right)$.

Proof of Proposition 3. In this construction, each particle will be two-sided: the $S$-side will represent the color of the particle in the standard coupling, and the $M$-side the color in the modified standard coupling. A particle will be called a hybrid if the colors of its two sides disagree. The strategy will be to show that colors can be assigned in such a way that the process of hybrid particles is dominated by the green particle process of Corollary 1 the result (37) will then follow from (33).

Consider first the $S I S$ case. Observe that in this case $\kappa_{N}(y)$ is the conditional expectation, in the standard coupling, of the number of pairs that share labels, given that there are $y$ offspring of 
red parents at a site. Thus, $\kappa_{N}(y)$ exceeds (by a small amount) the conditional probability in the standard coupling that at least one of the $y$ red-parent offspring would be blue. Denote by $\Delta_{N}(y)$ the excess; note that

$$
\Delta_{N}(y)=O\left(y^{4} / N^{2}\right) .
$$

The rules by which the process evolves are as follows: First, all particles reproduce, each creating a random number of offspring with Poisson-1 distribution. Each offspring then moves $+1,-1$, or 0 steps from its birth site, and chooses a random label $j \in[N]$. Particles with "genotype" $B B$ (that is, offspring of particles with coloring $B B$; the first letter denotes the $S$-color, the second the $M$-color) will always be colored $B B$, and their labels $j$ will play no role in determining the colors of the other offspring. However, the labels of all other offspring matter. Say that there is an $S$-duplication at label $j$ if at least two particles both with a red $S$-gene choose label $j$; similarly, say that an $M$-duplication occurs at $j$ if $j$ is chosen by at least two particles with $M$-gene $R$. (Note: If both $B R$ and $R B$ genotype particles choose label $j$, only the $R B$ particles are counted in the possible $S$-duplication, and only $B R$ particles in the $M$-duplication.) Particles at $(x, t)$ are now assigned color "phenotypes" by the following rules:

(D) (Default) If there is a duplication involving at least two particles of genotype $R R$, do the following: Among all such duplications, choose one (say $i$ ) at random; choose one of the genotype- $R R$ particles with label $i$, assign it phenotype $B B$, and give all of the other particles with label $i$ the same genotypes as their parents. Give all other particles at the site the same $M$-colors as their parents, and assign the remaining $S$-colors by rule (S):

(S) If there are labels $j \neq i$ with $S$-duplications, then for each such label

(a) If there is at least one particle with genotype $R B$ in the duplication, then choose one of all such $R B$ particles at random, give it phenotype $B B$, and give all other particles involved in the duplication $S$-color $R$.

(b) Otherwise, if all particles involved in the duplication have genotype $R R$, choose one at random and assign it phenotype $B R$, and give all of the rest phenotype $R R$.

(c) Give all particles not involved in $S$-duplications the same $S$-colors as their parents.

(M) If there are no duplications of type (D) but at least one $M$-duplication, then in any such duplication, at least one particle of genotype $B R$ must be involved. Choose one at random and assign it phenotype $B B$, give all of the remaining particles at the site $M$-color $R$, and assign $S$-colors by rules (S)-(a),(c).

(A) (Adjustment Step) If there are no $M$-duplications, then toss a $\Delta_{N}(y)-$ coin: If it comes up Heads, choose one of the particles with genotype ? $R$ at random, give it $M$-color $B$, and give all of the rest $M$-color $R$. If it comes up Tails, give every particle the same $M$-color as its parent.

These rules guarantee that the $S$-colors of the particles behave as in the standard coupling, and that the $M$-colors behave as in the modified standard coupling. Therefore, the discrepancy $Y_{t}^{N, \Delta}(x)$ is bounded by the number of hybrid particles at $(x, t)$. Hybrids can be offspring of $R R$, $B R$, or $R B$ particles, but not $B B$ particles; however, a hybrid can only be produced by a particle of type $R R$ if (i) there is a multiple collision, i.e., if there are at least two pairs of non- $B B$ particles that choose the same labels; or (ii) the coin toss in the adjustment step (A) is a Head. Both of these are events of (conditional) probability $O\left(y^{4} / N^{2}\right)$. Moreover, by Lemma 3, except with vanishingly small probability, there is no site/time $(x, t)$ with more than 3 hybrid offspring of $R R$ parents. Consequently, on the event that the maximal number of particles at any site/time is no more than $C N^{\alpha / 2}$ (see (28) above), the process of $R R \rightarrow$ hybrid creations is dominated as follows: Let each particle, in every generation, produce an additional Poisson- $2 C N^{3 \alpha / 2-2}$ offspring; immediately replace each such particle by 3 green particles, and let green particles only beget other green particles 
in subsequent generations. Since $\alpha \leq 2 / 3$, the rate at which green particles are produced by nongreen particles is $O\left(N^{-1}\right)$, so Corollary 1 implies that the green particle process is asymptotically negligible. This proves (37) in the $S I S$ case. The SIR case is proved by a very similar construction.

The upshot of Proposition 3 is that in proving Theorem 1 the epidemic process (the red process in the extended standard coupling) can be replaced by the red process in the modified standard coupling.

2.5. Orthogonal martingale measures and convergence of stochastic integrals. Let $Y_{t}^{k}(x)$ be the number of particles at site $x \in \mathbb{Z}$ at time $[t] \in \mathbb{Z}_{+}$in a one-dimensional branching random walk with Poisson-1 offspring law $\mathcal{R}_{\infty}$. Denote by $X_{t}^{k}=\mathcal{F}_{k} Y_{k t}^{k}$ the corresponding rescaled measurevalued process. For each $k$, the measure-valued process $X_{t}^{k}$ satisfies a martingale problem analogous to that satisfied by the super-Brownian motion: If $\phi \in C_{b}^{\infty}$, the (cadlag) process

$$
M_{t}^{k}(\phi):=\left\langle X_{t}^{k}, \phi\right\rangle-\left\langle X_{0}^{k}, \phi\right\rangle-\int_{0}^{[k t] / k}\left\langle X_{s}^{k}, A_{k} \phi\right\rangle d s
$$

is a martingale, where $A_{k}$ is the difference operator

$$
\left.A_{k} \phi(x)=\{\phi(x+1 / \sqrt{k})+\phi(x-1 / \sqrt{k})-2 \phi(x))\right\} / 3 k^{-1} .
$$

(Since $X_{t}$ is constant on successive time intervals of duration $k^{-1}$, the integral in (39) is really a sum.) The operator $\phi \mapsto M^{k}(\phi)$ extends to an orthogonal martingale measure $M^{k}(d s, d x)$ (see 23 for the definition and basic stochastic integration theory). The measure $M^{k}$ is purely discrete, putting mass only at points $(s, x) \in k^{-1} \mathbb{Z}_{+} \times k^{-1 / 2} \mathbb{Z}$ : at such points $s=n / k, x=m / \sqrt{k}$,

$$
\begin{aligned}
k M^{k}(\{s\},\{x\}) & =Y_{n}^{k}(m)-\lambda_{n}^{k}(m) \quad \text { where } \\
\lambda_{n}^{k}(m) & =\lambda^{k}(s, x):=E\left(Y_{n}^{k}(m) \mid \mathcal{H}_{n-1}\right)=\frac{1}{3} \sum_{i=0,-1,1} Y_{n-1}^{k}(x+i), \quad \text { and } \\
\mathcal{H}_{n} & =\sigma\left(\left\{Y_{j}^{k}(m): m \in \mathbb{Z}, j \leq n\right\}\right)
\end{aligned}
$$

is the $\sigma$-algebra generated by the history of the evolution to time $n$. Note that mass is scaled by the factor $k$, as required by the Feller-Watanabe normalization. Note also that, conditional on $\mathcal{H}_{n-1}$, the random variables $\left\{Y_{n}^{k}(m)\right\}_{m \in \mathbb{Z}}$ are mutually independent: this implies that the martingale measure $M^{k}$ is an orthogonal martingale measure.

Proposition 4. Assume that the initial particle densities $X^{k}(0, \cdot)$ satisfy the hypotheses of Theorem Q, that is, they have common compact support and they converge in $C_{b}(\mathbb{R})$ to a continuous function $X(0, \cdot)$. Then the random vectors $\left(X^{k}, M^{k}\right)$ consisting of the density functions $X^{k}(t, x)$ and the orthogonal martingale measures $M^{k}$ converge weakly as $k \rightarrow \infty$ to $(X, M)$, where $X$ is the DawsonWatanabe density process (super-Brownian motion) with initial condition $X(0, x)$, and $M$ is its associated orthogonal martingale measure.

Proof. Consider first the marginal distributions of the orthogonal martingale measures $M^{k}$, viewed as random elements of the Skorohod space $\mathbb{D}\left([0, \infty), \mathcal{S}^{\prime}\right)$, where $\mathcal{S}^{\prime}$ is the space of tempered distributions on $\mathbb{R}$. In order to prove that $M^{k} \Rightarrow M$ it suffices, by Mitoma's theorem (cf. 23, Th. 6.15), to prove that (i) for any $\phi \in \mathcal{S}$ (where $\mathcal{S}$ is the Schwartz space of test functions), the processes $M_{t}^{k}(\phi)$ are tight, and (ii) finite-dimensional distributions $M_{t_{i}}^{k}\left(\phi_{i}\right)$ converge for all $\phi_{i} \in \mathcal{S}$. Both of these follow routinely from the representation (39) and Watanabe's theorem: In particular, Watanabe's theorem implies that for any finite subset $\left\{\phi_{i}\right\}_{i \in I}$ of $\mathcal{S}$,

$$
\left(\left\langle X_{t}^{k}, \phi_{i}\right\rangle-\left\langle X_{0}^{k}, \phi_{i}\right\rangle\right)_{i \in I} \Longrightarrow\left(\left\langle X_{t}, \phi_{i}\right\rangle-\left\langle X_{0}, \phi_{i}\right\rangle\right)_{i \in I}
$$


and

where

$$
\left(\int_{0}^{t}\left\langle X_{s}^{k}, A \phi_{i}\right\rangle d s\right)_{i \in I} \Longrightarrow\left(\int_{0}^{t}\left\langle X_{s}, \phi_{i}\right\rangle d s\right)_{i \in I}
$$

$$
A=\lim _{k \rightarrow \infty} A_{k}=\Delta / \sqrt{6}
$$

Consequently, to deduce (i)-(ii) above it suffices to show that for each $\phi=\phi_{i}$,

$$
\int_{0}^{t}\left\langle X_{s}^{k},\left|A_{k} \phi-A \phi\right|\right\rangle d s+\int_{[k t] / k}^{t}\left\langle X_{s}^{k},|A \phi|\right\rangle d s \stackrel{P}{\longrightarrow} 0 .
$$

Since $\left\|A_{k} \phi-A \phi\right\|_{\infty} \rightarrow 0$ for Schwartz-class functions $\phi$, this also follows from Watanabe's theorem.

It remains to show that the convergence $M^{k} \Rightarrow M$ holds jointly with $X^{k}(t, x) \Rightarrow X(t, x)$. Since $X^{k} \Rightarrow X$ (Theorem 2) and $M^{k} \Rightarrow M$ marginally, the joint distributions are tight. Hence, to prove that $\left(X^{k}, M^{k}\right)$ converge jointly, it suffices to show that the only possible weak limit is $(X, M)$, where $X=X(t, x)$ is the density process associated with the Dawson-Watanabe process. But because a continuous function $w(x)$ is determined by its integrals against Schwartz-class functions $\phi$, it suffices to show that finite dimensional distributions of the vector-valued processes $\left(M_{t}^{k} \phi_{i},\left\langle X_{t}^{k}, \phi_{i}\right\rangle\right)_{i \in I}$ converge to the corresponding joint distributions of $\left(M_{t} \phi_{i},\left\langle X_{t}, \phi_{i}\right\rangle\right)_{i \in I}$. This follows by a repetition of the argument in the preceding paragraph.

Corollary 2. Let $\theta(t, x, w)$ be a bounded, jointly continuous function of $t \geq 0, x \in \mathbb{R}$, and $w \in$ $\mathbb{D}\left([0,0), C_{c}(\mathbb{R})\right)$ such that for any $t$ the function $\theta(t, x, w)$ depends only on $w[0, t]$, that is, $\theta(t, x, w)=$ $\theta(t, x, w[0, t])$. Assume that the hypotheses of Proposition 4 hold. Then

$$
\iint \theta\left(s, x, X^{k}\right) M^{k}(d s, d x) \Longrightarrow \iint \theta(s, x, X) M(d s, d x)
$$

Moreover, (44) holds jointly with the convergence $X^{k} \Rightarrow X$ in $\mathbb{D}\left([0, \infty), C_{b}(\mathbb{R})\right)$.

Proof. This can be deduced from Prop. 7.6 of 23 , but verification of the hypothesis (7.5) is more work than a direct proof. The elementary Prop. 7.5 of 23 implies that weak convergence (44) (and joint convergence with $X^{k} \Rightarrow X$ ) holds for simple integrands

$$
\theta(t, x, w)=\sum_{i=1}^{n} a_{i}(w) \mathbf{1}_{\left(s_{i}, t_{i}\right]}(t) \phi_{i}(x)
$$

such that (a) each $\phi_{i} \in \mathcal{S}$, (b) each $a_{i}(w)$ is bounded, continuous in $w$, and $\mathbb{F}_{s_{i}}$-measurable (here $\left(\mathbb{F}_{s}\right)$ is the natural filtration on $\left.\mathbb{D}\left([0, t], C_{c}(\mathbb{R})\right)\right)$, and (c) none of the jump times $s_{i}, t_{i}$ coincides with jumps of one of the martingale measures $M^{k}$. Clearly, any function $\theta$ satisfying the hypotheses of Corollary 2 can be uniformly approximated by such simple functions. Consequently, to prove (44) it suffices to show that for any $\varepsilon>0$ there exists $\delta=\delta(\varepsilon)>0$ such that for any simple function $\theta$ satisfying (a)-(c) and $\|\theta\|_{\infty}<\delta$,

$$
P\left\{\left|\int \theta(t, x) M^{k}(d t, d x)\right|>\varepsilon\right\}<\varepsilon \quad \forall k .
$$

To establish (45) we use the special structure of the orthogonal martingale measure $M^{k}$. For each $k$, this is a purely discrete random measure with atoms (411). By hypothesis, the conditional distribution of $Y_{n}^{k}(m)$ given the past is Poisson with mean (and therefore also variance) $\lambda_{n}^{k}(m)$ (see (42)), and the random variables $\left\{Y_{n}^{k}(m)\right\}_{m \in \mathbb{Z}}$ are, for each fixed $n$, conditionally independent given the past. Hence, the predictable quadratic variation of the local martingale

$$
\left(\theta \cdot M^{k}\right)_{t}:=\int_{0}^{t} \int_{x} \theta(s, x) M^{k}(d s, d x)
$$


is

$$
\left[\theta \cdot M^{k}\right]_{t}=\sum \sum \mathbf{1}_{[0, t)}(s) \theta(s, x)^{2} \lambda^{k}(s, x) / k^{2},
$$

where the sum is over the jump points $(s, x) \in k^{-1} \mathbb{Z}_{+} \times k^{-1 / 2} \mathbb{Z}$ of the martingale measure $M^{k}$. Thus, if $\|\theta\|_{\infty}<\delta$, then the quadratic variation of $\left(\theta \cdot M^{k}\right)_{t}$ is bounded by

$$
\delta^{2} \sum \sum \lambda^{k}(s, x) / k^{2}=\delta^{2} \sum \sum Y^{k}(s, x) / k^{2}
$$

But $\sum \sum Y^{k}(s, x) / k^{2}$ is just the total rescaled mass in the branching random walk, which by Watanabe's theorem (or Feller's theorem) converges in law to the total mass in the standard DawsonWatanabe process. The inequality (45) now follows routinely.

Unfortunately, the functions for which we would like to apply this result - namely, those defined by equations (14) and (13) - are unbounded. Worse, the function $\theta(t, x)=X(t, x)$ in equation (13) isn't even continuous (as a function of $X(t, x) \in \mathbb{D}\left([0, \infty) \times C_{c}(\mathbb{R})\right.$ ), because such functions may have jumps). The following corollary takes care of the first problem.

Corollary 3. Assume that the hypotheses of Proposition 母 are satisfied. Let $\theta(t, x, w)=\theta(t, x, w[0, t])$ be a jointly continuous function of $t \geq 0, x \in \mathbb{R}$, and $\left(w \in \mathbb{D}\left([0, \infty), C_{c}(\mathbb{R})\right)\right.$ such that for every scalar $C<\infty$ and every compact subset $F$ of $[0, \infty) \times \mathbb{R}$,

$$
\sup \left\{|\theta(t, x, w)|: \sup _{t^{\prime}, x^{\prime}}\left|w\left(t^{\prime}, x^{\prime}\right)\right| \leq C \text { and } \operatorname{support}(w) \subset F\right\}<\infty .
$$

Then (44) holds jointly with the convergence $X^{k} \Rightarrow X$ in $\mathbb{D}\left([0, \infty), C_{b}(\mathbb{R})\right)$.

Observe that the hypothesis is satisfied by the function $\theta$ defined by (14).

Proof. Because the Dawson-Watanabe density process $X(s, x)$ is almost surely continuous with compact support, continuity of $\theta$ ensures that

$$
\iint \theta(s, x, X)^{2} X(s, x) d s d x<\infty
$$

and this in turn guarantees that

$$
\lim _{C \rightarrow \infty} \iint(\theta(s, x, X) \wedge C) M(d s, d x)=\iint \theta(s, x, X) M(d s, d x)
$$

and that the limit is a.s. finite. By the hypothesis (46), the function $\theta$ is uniformly bounded on any set of sample evolutions $\omega=\left(x_{s}\right)$ such that $\operatorname{support}(\omega)$ and $\sup |\omega|$ are uniformly bounded. By the results of sec. 2.2 above, the supports and suprema of the random functions $X^{k}(t, x)$ are tight, that is, for any $\varepsilon>0$ there exist a compact set $F=F_{\varepsilon}$ and a constant $C=C_{\varepsilon}<\infty$ such that for all $k$,

$$
P\left\{\operatorname{support}\left(X^{k}\right) \subset F \text { and } \max X^{k}(t, x) \leq C\right\} \geq 1-\varepsilon .
$$

Consequently, since the support of the martingale measure $M^{k}$ is contained in that of $X^{k}$, it follows that for any $\varepsilon>0$ there exists $C<\infty$ such that for all $k$,

$$
\iint \theta(s, x, X) M^{k}(d s, d x)=\iint(\theta(s, x, X) \wedge C) M^{k}(d s, d x)
$$

except on a set of probability $\leq \varepsilon$. Weak convergence (44) (and joint weak convergence with $X^{k} \Rightarrow X$ ) now follows routinely from Corollary 2 and (47).

The function $\theta(t, x)=X(t, x) / 2$ that occurs in the SIS case of Theorem 1 is not continuous for functions $X(t, x)$ in the space $\mathbb{D}\left([0, \infty), C_{c}(\mathbb{R})\right)$, because such functions may have jumps. Thus, Corollaries 23 do not apply directly. The following corollary addresses this case specifically. 
Corollary 4. Assume that the hypotheses of Proposition 4 are satisfied, and define $\theta^{k}(s, x)=$ $\lambda^{k}(s, x) / \sqrt{k}$, where $\lambda^{k}(s, x)$ is as in (42) above. Then

$$
\iint \theta^{k}(s, x) M^{k}(d s, d x) \Longrightarrow \iint X(s, x) M(d s, d x),
$$

and this convergence holds jointly with $X^{k} \Rightarrow X$.

Proof. Although the random functions $X^{k}(t, x)$ and $\lambda^{k}(t, x)$ have jumps, the jumps are, with high probability, small, because $X^{k}(t, x) \Rightarrow X(t, x)$, by Theorem 2. Consequently, it is still possible to approximate $\theta^{k}$ by bounded, continuous functions $\varphi$ in such a way that the stochastic integrals of $\theta^{k}$ relative to $M^{k}$ are well approximated, with high probability, by those of $\varphi$. In particular, define, for any $\varepsilon>0$ and $C<\infty$,

$$
\varphi(s, x)=\varphi_{C, \varepsilon}(s, x, w)=\frac{1}{2 \varepsilon^{2}} \iint_{\substack{s^{\prime} \in[s-2 \varepsilon, s-\varepsilon] \\ x^{\prime} \in[x-\varepsilon, x+\varepsilon]}} w\left(s^{\prime}, x^{\prime}\right) \wedge C d s^{\prime} d x^{\prime} ;
$$

then for any $\delta>0$ there exist $C, \varepsilon$ such that for all $k$,

$$
P\left\{\max _{s, x}\left|\theta^{k}(s, x)-\varphi\left(s, x, X^{k}\right)\right|>\delta\right\}<\delta .
$$

Clearly, $\varphi(s, x, w)$ is jointly continuous in its arguments and uniformly bounded by $C$, so it meets the requirements of Corollary 2, It follows that for any $C, \varepsilon$,

$$
\left.\iint \varphi\left(s, x, X^{k}\right) M^{k}(d s, d x) \Longrightarrow \iint \varphi(s, x, X)\right) M(d s, d x),
$$

and this holds jointly with $X^{k} \Rightarrow X$. Thus, to prove the corollary, it suffices to show that if $C$ and $\varepsilon$ are suitably chosen then the differences between the stochastic integrals of $\theta^{k}$ and $\varphi$ against the martingale measures $M^{k}$ are small with high probability, uniformly in $k$.

By virtually the same calculation as in the proof of Corollary 3, the local martingale

$$
\iint_{s \leq t}\left(\theta^{k}(s, x)-\varphi\left(s, x, X^{k}\right)\right) M^{k}(d s, d x):=\iint_{s \leq t} \Delta^{k}(s, x) M^{k}(d s, d x)=\left(\Delta^{k} \cdot M^{k}\right)_{t}
$$

has predictable quadratic variation

$$
\begin{aligned}
{\left[\Delta^{k} \cdot M^{k}\right]_{t} } & =\sum \sum \mathbf{1}_{[0, t)}(s) \Delta^{k}(s, x)^{2} \lambda^{k}(s, x) / 2 \\
& \leq \max _{s, x} \Delta^{k}(s, x)^{2} \sum \sum Y^{k}(s, x) / k^{2} .
\end{aligned}
$$

If $\varepsilon>0$ is sufficiently small and $C<\infty$ sufficiently large, then by inequality (49), $\left\|\Delta^{k}\right\|_{\infty}<\delta$ except with small probability, uniformly for all $k$. Recall that the sum $\sum \sum Y^{k}(s, x) / k^{2}$ is the total rescaled mass in the branching process, and so by Feller's theorem converges in law to the total mass in the standard Dawson-Watanabe process. Hence, with high probability, the quadratic variation $\left[\Delta^{k} \cdot M^{k}\right]_{\infty}$ will be small, provided $C$ and $\varepsilon$ are chosen so that $\delta$ is small. Therefore, by standard martingale arguments, the maximum modulus of the stochastic integral $\left(\Delta^{k} \cdot M^{k}\right)_{t}$ will be small, with high probability, uniformly in $k$.

2.6. Likelihood ratios: generalities. The strategy of the proof of Theorem 1 is to show that the likelihood ratios of the (modified) spatial epidemic processes relative to their branching envelopes converge weakly to the likelihood ratios of the appropriate Dawson-Watanabe processes with killing relative to the Dawson-Watanabe process with no killing. The likelihood ratio of the DawsonWatanabe process with killing is given by (10). This expression involves a stochastic integral relative to the orthogonal martingale measure of a standard Dawson-Watanabe process, and so to prove weak 
convergence we will express the likelihood ratios of the epidemic processes in terms of stochastic integrals.

Consider a sequence $Y_{t}^{N}(x)$ of counting processes, and probability measures $P=P^{N}, Q=Q^{N}$ such that under $P$ the process $Y^{N}$ is a branching random walk with offspring law $\mathcal{R}_{\infty}$, and under $Q$ it is a modified epidemic process (that is, the red particle process in the modified standard coupling of sec. 2.4). Assume that the initial conditions $Y_{0}^{N}$ are common under $P$ and $Q$, and satisfy the hypotheses of Theorem [1. Let $\mathcal{H}_{t}$ be the $\sigma$-algebra generated by the history of the evolution to time $t$, and set

$$
\begin{aligned}
\lambda_{t}^{N}(x): & =E_{P}\left(Y_{t}^{N}(x) \mid \mathcal{H}_{t-1}\right) \\
& =\left(Y_{t-1}^{N}(x-1)+Y_{t-1}^{N}(x)+Y_{t-1}^{N}(x+1)\right) / 3 .
\end{aligned}
$$

Under $P$, the random variables $Y_{t}^{N}(x)$ are, for each $t$, conditionally independent Poisson r.v.s, given $\mathcal{H}_{t-1}$, with conditional means $\lambda_{t}^{N}(x)$. In the modified standard couplings, the color choices at the various sites $x$ are, conditional on $\mathcal{H}_{t-1}$ and on the numbers of red-parent offspring at the various sites, mutually independent, with at most one blue offspring of a red parent at any site/time. Hence, the event $Y_{t}^{N}(x)=y$ could occur in one of only two ways: (1) there are $y$ offspring of red parents, none of which takes the color blue; or (2) there are $y+1$ offspring of red parents, one of which is blue. It follows that the relative likelihood $L_{N}:=d Q^{N} / d P^{N}$ of a sample evolution $\left(y_{t}(x)\right)_{t, x}$ is given by

$$
L_{N}=\prod_{t \geq 1} \prod_{x \in \mathbb{Z}} L_{N}(t, x):=\prod_{t \geq 1} \prod_{x \in \mathbb{Z}} \frac{p(y \mid \lambda)\left(1-\kappa_{N}(y)\right)+p((y+1) \mid \lambda) \kappa_{N}(y+1)}{p(y \mid \lambda)}
$$

where in each factor, $y=y_{t}(x)$ and $\lambda=\lambda_{t}^{N}(x)$, and $\kappa_{N}(y)=\kappa_{N, t, x}(y)$ is the conditional probability in the modified coupling, given $y$ offspring of red parents at $(t, x)$, that one of these is colored blue. Here $p(\cdot \mid \lambda)$ is the Poisson density with parameter $\lambda$. The conditional probability $\kappa_{N}(y)$ is given by (34) for SIS epidemics, and by (35) for SIR epidemics.

Although the product (51) extends over infinitely many sites and time $\mathrm{x}, \mathrm{t}$, all but finitely many of the factors are 1: In particular, if the nearest neighbors (in the previous generation) $\left(x^{\prime}, t-1\right)$ of site $(x, t)$ are devoid of particles, then the factor $L_{N}(t, x)$ indexed by $(x, t)$ must be 1 . Recall (by (23) and (27) of sec. 2.2) that under the hypotheses of Theorem 1, the number of site/time pairs $(x, t)$ visited by the branching envelope is of order $O_{P}\left(N^{3 \alpha / 2}\right)$. Thus, the number of nontrivial factors in (51) is typically of the same order.

2.7. Likelihood ratios: $\boldsymbol{S I R}$ epidemics. Analysis of the likelihood ratios is somewhat easier in the $S I R$ case, in that the error terms are more easily disposed. This is because in the SIR case Theorem 1 requires that $\alpha \leq 2 / 5$, and so the number of sites that contribute to the product (51) is $O_{P}\left(N^{3 / 5}\right)$. In particular, error terms of order $o_{P}\left(N^{-3 / 5}\right)$ can be ignored.

Recall that in the modified standard coupling for the $S I R$ epidemic, the conditional probability $\kappa_{N}(y)=\kappa_{N, t, x}(y)$ that there is a blue offspring of a red parent at $(t, x)$ is $y R / N$, where $R=R_{t}^{N}(x)$ is the number of labels used previously at site $x$ (see (36) ). Hence, the contribution to the likelihood ratio from the site $(t, x)$, on the sample evolution $Y_{t}^{N}(x)=y_{t}(x)$, is

$$
\begin{aligned}
L_{N}(t, x) & =1-y R / N+(\lambda /(y+1))(y+1) R / N \\
& =1-(y-\lambda) R / N .
\end{aligned}
$$


Thus, in the $S I R$ case, the likelihood ratio (51) can be written as

$$
\begin{aligned}
L_{N} & =\prod_{t} \prod_{x}\left(1-\left(\left(Y^{N}-\lambda_{t}^{N}(x)\right) R_{t}^{N}(x) / N\right)\right. \\
& =\prod_{t} \prod_{x} \exp \left\{\log \left(1-\Delta_{t}^{N}(x) \varrho_{t}^{N}(x)\right)\right\} \\
& =\left(1+\varepsilon_{N}\right) \exp \left\{-\sum_{t} \sum_{x} \Delta_{t}^{N}(x) \varrho_{t}^{N}(x)-\frac{1}{2} \sum_{t} \sum_{x} \Delta_{t}^{N}(x)^{2} \varrho_{t}^{N}(x)^{2}\right\},
\end{aligned}
$$

where

$$
\begin{aligned}
\Delta_{t}^{N}(x) & =\left(Y_{t}^{N}(x)-\lambda_{t}^{N}(x)\right) / N^{\alpha}, \\
\varrho_{t}^{N}(x) & =R_{t}^{N}(x) / N^{1-\alpha}, \quad \text { and } \\
\varepsilon_{N} & =o_{P}(1) .
\end{aligned}
$$

(Note: The error in the two-term Taylor series approximation to the logarithm is of magnitude $O_{P}\left(Y^{3} R^{3} / N^{3}\right)=O_{P}\left(N^{9 \alpha / 2-3}\right)$, which is asymptotically negligible for $\alpha \leq 2 / 5$; hence (56).)

The two sums that occur in the last exponential in (53) are a stochastic integal and its corresponding quadratic variation, respectively. To see this, observe that the quantities $\Delta_{t}^{N}(x)$ coincide with the masses (41) in the orthogonal martingale measures $M^{N}$ associated with the branching random walks $Y^{N}$. (In (41), $k=N^{\alpha}$; it makes more sense here to index by $N$ rather than $k$.) Consequently,

$$
\sum_{t} \sum_{x} \Delta_{t}^{N}(x) \varrho_{t}^{N}(x)=\iint \theta^{N}(t, x) M^{N}(d t, d x)
$$

where

$$
\theta^{N}(t, x)=\varrho_{t N^{\alpha}}^{N}\left(x N^{\alpha / 2}\right)=N^{5 \alpha / 2-1} \int_{s=0}^{t} X^{N}(s, x) d s
$$

For $\alpha<2 / 5$, this converges to zero as $N \rightarrow \infty$; for $\alpha=2 / 5$ it coincides with $\theta(t, x)=\theta\left(t, x, X^{N}\right)$ as given by (14). Therefore, Corollary 3 implies that

$$
\iint \theta^{N}(t, x) M^{N}(d t, d x) \Longrightarrow \iint \theta(t, x) M(d t, d x)
$$

where $M$ is the orthogonal martingale measure attached to the standard Dawson-Watanabe process and $\theta(t, x)$ is as in parts (c)-(d) of Theorem 1. (Corollary 3 also implies that the convergence holds jointly with $X^{N} \Rightarrow X$.) Consequently, to complete the proof that

$$
L_{N} \Longrightarrow L=\exp \left\{\iint \theta(t, x) M(d t, d x)-\frac{1}{2} \iint \theta(t, x)^{2} X(t, x) d t d x\right\}
$$

it suffices to prove that

$$
\sum_{t} \sum_{x} \Delta_{t}^{N}(x)^{2} \varrho_{t}^{N}(x)^{2} \Longrightarrow \iint \theta(t, x)^{2} X(t, x) d t d x
$$

Proof of (60). This uses only Theorem 2 and a variance calculation. Define

$$
\begin{aligned}
& A^{N}=\sum_{t} \sum_{x} \Delta_{t}^{N}(x)^{2} \varrho_{t}^{N}(x)^{2} \quad \text { and } \\
& B^{N}=\sum_{t} \sum_{x} \lambda_{t}^{N}(x) \varrho_{t}^{N}(x)^{2} / N^{2 \alpha} ;
\end{aligned}
$$


we will show that

$$
A^{N}-B^{N}=o_{P}(1)
$$

Since $\lambda_{t N^{\alpha}}^{N}\left(x N^{\alpha / 2}\right) / N^{\alpha / 2}-X^{N}(t, x)=o_{P}(1)$ as $N \rightarrow \infty$, by Theorem 2 (recall (50) that $\lambda^{N}(t, x)$ is the average of the counts $Y_{t-1}^{N}\left(x^{\prime}\right)$ over the neighboring sites $\left.x^{\prime}=x, x \pm 1\right)$,

$$
B^{N}=\iint X^{N}(t, x) \theta^{N}(t, x)^{2} d t d x+o_{P}(1) .
$$

Theorem 2 also implies that

$$
\iint X^{N}(t, x) \theta^{N}(t, x)^{2} d t d x \Longrightarrow \iint X(t, x) \theta(t, x)^{2} d t d x
$$

Therefore, proving (63) will establish (60).

For any constant $C<\infty$, define

$$
\tau_{C}=\tau_{C}^{N}=\max \left\{t: \max _{x} \max _{s \leq t} Y_{s-1}^{N}(x) \leq C N^{\alpha / 2}\right\},
$$

and let $A_{C}^{N}$ and $B_{C}^{N}$ be the restrictions of the sums (61)- (62) to the range $t \leq \tau_{C} \wedge C N^{\alpha}$ and $|x| \leq C N^{\alpha / 2}$. Note that $\tau_{C}$ is a stopping time, and that $\lambda_{t}^{N}(x) \leq C N^{\alpha / 2}$ on the event $t \leq \tau_{C}$. Since the range of summation in (61) and (62) is limited by (23) and (27), for any $\varepsilon>0$ there exists $C=C_{\varepsilon}<\infty$, independent of $N$, such that

$$
A^{N}=A_{C}^{N} \quad \text { and } \quad B^{N}=B_{C}^{N}
$$

except possibly on events of probability $\leq \varepsilon$. Thus, it suffices to prove (63) with $A^{N}, B^{N}$ replaced by $A_{C}^{N}, B_{C}^{N}$. For this, we use the fact that the offspring counts $Y_{t}^{N}(x)$ have conditional Poisson distributions (given the past $\mathcal{H}_{t-1}$ ) with means $\lambda_{t}^{N}(x)$ ): This implies that the conditional means and conditional variances coincide, and that

$$
E_{P}\left(\left(\left(Y_{t}^{N}(x)-\lambda_{t}^{N}(x)\right)^{2}-\lambda_{t}^{N}(x)\right)^{2} \mid \mathcal{H}_{t-1}^{N}\right)=2 \lambda_{t}^{N}(x)^{2} .
$$

For $t \leq \tau_{C}$, the right side is bounded by $2 C^{2} N^{\alpha}$. Furthermore, for $t \leq \tau_{C} \wedge C$,

$$
\varrho_{t}^{N}(x)^{4} \leq C^{8} N^{2 \alpha} / N^{4-4 \alpha} .
$$

Thus, the conditional variance of each term in the sum $A_{C}^{N}-B_{C}^{N}$ is bounded by $2 C^{10} N^{7 \alpha-4}$. Since the number of nonzero terms in the sum is $C(2 C+1) N^{3 \alpha / 2}$, it follows that

$$
E\left(A_{C}^{N}-B_{C}^{N}\right)^{2} \leq C^{\prime} N^{17 \alpha / 2-4} .
$$

But $\alpha \leq 2 / 5$, so this converges to 0 as $N \rightarrow \infty$.

2.8. Likelihood ratios: $\boldsymbol{S I S}$ epidemics. For $S I S$ epidemics, Theorem 1 requires that $\alpha \leq 2 / 3$, so by (23) and (27), the number of sites/times $(x, t)$ that contribute nontrivially to the likelihood ratio product (51) is $O_{P}\left(N^{3 \alpha / 2}\right)=O_{P}(N)$. Thus, error terms of magnitude $o_{P}\left(N^{-1}\right)$ can be ignored in each factor.

In the modified standard couplings for SIS epidemics, the conditional probability that there is a blue offspring of a red parent at $(x, t)$, given $y$ red-parent offspring in total at $(x, t)$, is $\kappa_{N}(y)=$ $y(y-1) / 2 N($ see $(34))$. Hence,

$$
\begin{aligned}
L_{N}(t, x) & =1-\left(\begin{array}{c}
y \\
2
\end{array}\right) / N+\frac{\lambda}{y+1}\left(\begin{array}{c}
y+1 \\
2
\end{array}\right) / N \\
& =1-y(\lambda-y-1) / 2 N \\
& =1-\lambda(y-\lambda) / 2 N-(y-\lambda)^{2} / 2 N+y / 2 N .
\end{aligned}
$$


Since terms of order $o_{P}\left(N^{-1}\right)$ can be ignored, (51) can be written as

$$
L_{N}=\left(1+o_{P}(1)\right) \prod_{t \geq 1} \prod_{x \in \mathbb{Z}} \exp \left\{-A_{N}(t, x)-B_{N}(t, x)-C_{N}(t, x) / 2\right\}
$$

where

$$
\begin{aligned}
& A_{N}(t, x)=\lambda(y-\lambda) / 2 N ; \\
& B_{N}(t, x)=\lambda^{2}(y-\lambda)^{2} / 8 N^{2} ; \\
& C_{N}(t, x)=(y-\lambda)^{2} / N-y / N .
\end{aligned}
$$

Here we continue to use the convention $y=y_{t}(x)$ and $\lambda=\lambda_{t}^{N}(x)$, as in (51). Hence, to prove the convergence (59) $L_{N} \Rightarrow L$ (jointly with that of $X^{N}(t, x) \Rightarrow X(t, x)$ ), it will suffice to show that

$$
\begin{gathered}
\sum_{t, x} A_{N}(t, x) \Longrightarrow \iint \theta(t, x) M(d t, d x) ; \\
\sum_{t, x} B_{N}(t, x) \Longrightarrow \iint \theta(t, x)^{2} X(t, x) d t d x ; \text { and } \\
\sum_{t, x}\left(C_{N}(t, x)(t, x)\right) \Longrightarrow 0
\end{gathered}
$$

where $\theta(t, x)=0$ for $\alpha<2 / 3$ and $\theta(t, x)=X(t, x) / 2$ for $\alpha=2 / 3$, as in parts (a)-(b) of Theorem 1,

Proof of (66). This is virtually identical to the proof of the analogous convergence in the SIR case. The increments $\Delta_{t}^{N}(x):=\left(Y_{t}^{N}(x)-\lambda_{t}^{N}(x)\right) / N^{\alpha}$ are the masses (41) in the orthogonal martingale measures $M^{N}$, and so

$$
\sum_{t, x} A_{N}(t, x)=\iint \theta^{N}(t, x) M^{N}(d t, d x),
$$

where $\theta^{N}(t, x)=\lambda_{N^{\alpha} t}^{N}\left(x N^{\alpha / 2}\right) / 2 N^{1-\alpha}$. If $\alpha<2 / 3$ then $\max _{t, x} \theta^{N}(t, x) \rightarrow 0$ in probability, whereas if $\alpha=2 / 3$ then $\theta^{N}(t, x)$ meets the requirements of Corollary 4 . Thus, the convergence (66) follows from Corollary 4

Proof of (67). This proceeds in the same manner as the proof of (60) in the SIR case, by showing that the term $B_{N}(t, x)$ in the sum (67) can be replaced by $B_{N}^{\prime}(t, x):=\lambda^{3} / 8 N^{2}$. To do this, we truncate the sum of the differences in exactly the same way as in the proof of (60), using the same stopping times $\tau_{C}=\tau_{C}^{N}$. Note that the truncated sum once again has $C(2 C+1) N^{3 \alpha / 2}$ terms, and that in each term, $\lambda \leq C N^{\alpha / 2}$. Using the conditional variance formula (64), we find that after truncation,

$$
E\left(\sum \sum\left(B_{N}(t, x)-B_{N}^{\prime}(t, x)\right)\right)^{2} \leq C^{\prime} N^{3 \alpha / 2} N^{6 \alpha / 2} / N^{4} \leq C^{\prime} N^{-1}
$$

since $\alpha \leq 2 / 3$. Consequently, $\sum \sum B_{N}$ can be replaced by $\sum \sum B_{N}^{\prime}$. But

$$
\sum_{t} \sum_{x} B_{N}^{\prime}(t, x)=\sum_{t} \sum_{x} \lambda_{t}^{N}(x)^{3} / 8 N^{2} \Longrightarrow \iint X(t, x) \theta(t, x)^{2} d x d t
$$

by Theorem 2 and the definition of $\theta$ (parts (a)-(b) of Theorem 1)

Proof of (68). This is based on a variance calculation similar to those used to prove (67) and (60). Note first that the terms $C_{N}(t, x)$ constitute a martingale difference sequence relative to the filtration 
$\mathcal{H}_{t}$, because the mean and variance of a Poisson random variable coincide. The conditional variances of the terms $C_{N}(t, x)$ can be estimated as follows: If $Y \sim \operatorname{Poisson}(\lambda)$ with $\lambda \geq 1$, then

$$
\begin{aligned}
E\left((Y-\lambda)^{2}-Y\right)^{2} & =E\left((Y-\lambda)^{2}-\lambda\right)^{2}+E(Y-\lambda)^{2}+2 E\left((Y-\lambda)^{2}-\lambda\right)(Y-\lambda) \\
& \leq 2 \lambda^{2}+\lambda+2 \sqrt{2 \lambda^{2} \lambda} \\
& \leq 5 \sqrt{2} \lambda^{2} .
\end{aligned}
$$

Now truncate the sum $\sum \sum C_{N}(t, x)$ as before (that is, $t \leq \tau_{C} \wedge C N^{\alpha}$ and $|x| \leq C N^{\alpha / 2}$ ): Since the number of terms is $C^{\prime} N^{3 \alpha / 2}$ and $\lambda \leq C N^{\alpha / 2}$ for each term, the variance of the truncated sum is bounded by $C^{\prime \prime} N \lambda^{2} / N^{2}$. For $\alpha \leq 2 / 3$, this converges to zero as $N \rightarrow \infty$.

\section{Weak Convergence in $\mathbb{D}\left([0, \infty), C_{b}(\mathbb{R})\right)$ : Proof of Theorem 2}

In this section we prove Theorem 2 by verifying that the sequence of random functions $X^{M}(t, x)$ is tight, provided the hypotheses of Theorem 2 on the initial densities hold.

3.1. Moment Estimates. The proof will be based on moment estimates for occupation counts of a branching random walk started by a single particle located at the origin at time 0 . Denote by $Y_{n}(x)$ the number of particles at site $x \in \mathbb{Z}$ at time $n \in \mathbb{Z}_{+}$, and by $E^{x}, P^{x}$ the expectation operator and probability measure under which the branching random walk is initiated by a single particle located at $x$. For notational ease set

$$
\begin{aligned}
\varphi(x) & =\exp \left\{-x^{2}\right\}, \\
\varphi_{n}(x) & =\exp \left\{-x^{2} / n\right\}, \\
\Phi_{n}(x, y) & =\varphi_{n}(x)+\varphi_{n}(y) .
\end{aligned}
$$

Proposition 5. There exist finite constants $C_{m}, \beta_{m}$ such that for all $m, n \in \mathbb{N}$, all $x, y \in \mathbb{Z}$, and all $\alpha \in(0,1)$

$$
\begin{aligned}
E^{0} Y_{n}(x)^{m} & \leq C_{m} n^{-1} n^{m / 2} \varphi_{n}\left(\beta_{m} x\right), \\
\left|E^{0}\left(Y_{n}(x)-Y_{n}(y)\right)^{m}\right| & \leq C_{m} n^{-1} n^{m / 2}|(x-y) / \sqrt{n}|^{m / 5} \Phi_{n}(\beta x, \beta y), \\
\left|E^{0}\left(Y_{n}(x)-Y_{n+[\alpha n]}(x)\right)^{m}\right| & \leq C_{m} n^{-1} n^{m / 2} \alpha^{m / 5} \varphi_{n}\left(\beta_{m} x\right) .
\end{aligned}
$$

The (somewhat technical) proofs will be given in sections $3.5 / 3.7$ below. The exponents $m / 5$ on $\alpha$ and $|x-y| / \sqrt{n}$ in (72) and (71) are not optimal, but the orders of magnitude in the estimates make sense, as can be seen by the following reasoning: The probability that a branching random walk initiated by a single particle survives for $n$ generations is $O\left(n^{-1}\right)$, and on this event the total number of particles in the $n$th generation is $O(n)$. Thus, on the event of survival to generation $n$, the number of particles $Y_{n}(x)$ at a site $x$ at distance $\sqrt{n}$ from the origin should be $O(\sqrt{n})$. This is consistent with $m$ th moment of order $O\left(n^{-1} n^{m / 2}\right)$.

3.2. Tightness in $\mathbb{D}\left([\delta, \infty), C_{b}(\mathbb{R})\right)$. The proof of tightness will be broken into two parts: In this section, we will show that for any $\delta>0$, under the hypotheses of Theorem 2 , the density processes $X^{k}(t, x)$ restricted to the time interval $[\delta, \infty)$ are tight. Using this and an auxiliary smoothness result of Shiga 22 for the Dawson-Watanabe process, we will then conclude in sec. 3.3 that the density processes $X^{k}(t, x)$ with $t$ restricted to some interval $[0, \delta]$ are tight.

Standing Assumptions: In sections $3.2-3.3 Y_{t}^{k}(x)$ will be a sequence of branching random walks satisfying the hypotheses of Watanabe's theorem, and $X^{k}(t, x)$ will be the corresponding renormalized density processes, defined by equation (15). In addition, assume that the initial configurations 
are such that for every $k$, the initial particle density function $X^{k}(0, \cdot)$ has support contained in $J$, for some fixed compact interval $J$.

Proposition 6. There exist constants $C=C_{n}, p<\infty$ and $\beta>0$ such that for every $k=1,2, \ldots$, all $x, y \in \mathbb{R}$, and all $s, t \in[1 / n, n]$,

$$
\begin{aligned}
& E\left|X^{k}(t, x)-X^{k}(t, y)\right|^{p} \leq C|x-y|^{2+\delta}(\varphi(\beta x)+\varphi(\beta y)) \quad \text { and } \\
& E\left|X^{k}(t, x)-X^{k}(s, x)\right|^{p} \leq C|t-s|^{2+\delta} \varphi(\beta x) .
\end{aligned}
$$

Note. Under the conditions of Watanabe's theorem, the bounds (73)-(74) will in general hold only for $t, s$ away from zero: in fact, if $X_{0}$ is singular then the densities $X^{k}(t, x)$ will blow up as $t \rightarrow 0$.

Proof. The inequalities (73)-(74) follow from inequalities (71)-(72), respectively, with $p=m \geq 12$. To see this, observe that the moments in (73)-(74) can be related to the corresponding moments for branching random walks $Y$ started from single particles located at points $x \in J \sqrt{k}$ : If $n_{k}:=\left\|Y_{0}^{k}\right\|$ is the number of particles in the initial configuration of the $k$ th $B R W$ then for any even integer $m$,

$$
E\left|X^{k}(t, x)-X^{k}(t, y)\right|^{m}=\sum_{i=1}^{m} \sum_{\mathbf{m} \in \mathcal{P}_{r}(m)} \sum_{x_{1}, x_{2}, \ldots x_{r}} \prod_{i=1}^{r} k^{-m_{i} / 2} E^{x_{i}}\left(Y_{[t k]}(\sqrt{k} x)-Y_{[k t]}(\sqrt{k} y)\right)^{m_{i}}
$$

where $\mathcal{P}_{r}(m)$ is the set of all integer partitions of $m$ with $r$ nonzero elements $m_{i}$, the inner sum is over all choices of $r$ particles from the $n_{k}$ particles in the initial configuration, and $x_{i}$ is the location of the $i$ th particle. Since $\mathcal{F}_{k} Y_{0}^{k} \Rightarrow X_{0}$, the masses $n_{k}$ must be asymptotically proportional to $k$, so the number of choices in the inner sum is $\leq C k^{r}$, for some constant $C$ independent of $k$. Since the initial configurations are all restricted to lie in $\sqrt{k} J$, with $J$ compact, the bounds implied by (71) for $E^{x_{i}}$ are comparable to those for $E^{0}$, after changing $\beta_{m}$ to $\beta_{m} / 2$, because the Gauss kernel $\varphi$ satisfies $\varphi(x-y) \leq C_{J} \varphi(x / 2)$ for all $x \in \mathbb{R}$ and $y \in J$. Thus, by (71),

$$
R H S(75) \leq C \sum_{i=1}^{m} \sum_{\mathbf{m} \in \mathcal{P}_{r}(m)} k^{r} \prod_{i=1}^{r} k^{-1}|x-y|^{m_{i} / 5} \Phi_{1}(\beta x, \beta y),
$$

provided $t$ is bounded away from 0 and $\infty$. This clearly implies (73). A similar argument gives (74).

Corollary 5. Assume that all of the measures $\mathcal{F}_{k} Y_{0}^{k}$ have supports $\subset J$, where $J$ is compact, and assume that the hypothesis (7) of Watanabe's theorem holds. Then for every $\delta>0$, the random functions $\left\{X^{k}(t, x)\right\}_{t \geq \delta}$ converge weakly in the Skorohod space $\mathbb{D}\left([\delta, \infty), C_{b}(\mathbb{R})\right)$ to the Dawson-Watanabe density process $\{X(t, x)\}_{t \geq \delta}$ restricted to time $t \geq \delta$.

Remark 1. Observe that Corollary 5 holds even for initial conditions $Y_{0}^{k}(\cdot)$ whose Feller-rescalings $\mathcal{F}_{k} Y_{0}^{k}$ converge to singular measures.

Proof. Since the associated measure-valued processes converge to the Dawson-Watanabe process, it suffices to show that the sequence $\left\{X^{k}(t, x)\right\}_{t \geq \delta}$ is tight. This follows from inequalities (73)-(74) by the usual Kolmogorov-Chentsov argument. (See, for instance, 4], Th. 12.3, or [15, Problem 4.11 for the one-parameter case. Here, since there are two parameters $t, x$, the exponent must be $>2$.)

3.3. Tightness in $\mathbb{D}\left([0, \infty), C_{b}(\mathbb{R})\right)$. It remains to show that under the stronger hypotheses of Theorem 2 the rescaled particle densities $X^{k}(t, x)$ are tight for $t \in[0, \infty)$. It is possible to do this by estimating moments, but this is messy. Instead, we will use a soft argument, based on an estimate for the Dawson-Watanabe process proved by Shiga ([22, Lemma 4.2): 
Lemma 4. Let $X(t, x)$ be the density of a standard Dawson-Watanabe process with initial condition $X(0, x)=f(x)$ and variance parameter $\sigma^{2}$. Define

$$
N(t, x)=X(t, x)-G_{t} f(x)
$$

where $G_{t} f$ is the convolution of $f$ with the Gaussian density of variance $\sigma^{2} t$. For any compact interval $J$ there exist constants $C_{1}, C_{2}$ such that for all $\varepsilon, T>0$, if $f \leq \beta \mathbf{1}_{J}$ then

$$
P\left\{|N(t, x)| \geq \varepsilon \beta e^{-(T-t)|x|} \text { for some } t<T / 2 \text { and } x \in \mathbb{R}\right\} \leq C_{1} \varepsilon^{-24} \exp \left\{-C_{2} \varepsilon^{2} T^{-1 / 4}\right\} .
$$

Consequently, for any $\alpha>1, \varepsilon>0$, and compact interval $J$ there exists $T>0$ such that if $f \leq \beta \mathbf{1}_{J}$ then

$$
P\{X(t, x) \geq \alpha \beta \text { for some } t \leq T \text { and } x \in \mathbb{R}\}<\varepsilon .
$$

The strategy now is to use Lemma 4 to deduce a maximal inequality for the density $X^{k}(t, x)$ of a branching random walk over a short time interval $t \in[0, \delta]$. For this, we use the weak convergence result of Corollary $[5$ together with monotonicity of the $B R W$ in the initial condition. Note that adding particles to the initial configuration of a $B R W$ has the effect of augmenting the original $B R W$ by an independent $B R W$ initiated by the set of new particles. Thus, for any two initial particle configurations $Y_{0}^{k, A}$ and $Y_{0}^{k, B}$ whose discrepancy satisfies

$$
\left|Y_{0}^{k, A}(x)-Y_{0}^{k, B}(x)\right| \leq \beta k \mathbf{1}_{\sqrt{k} J}(x)
$$

there exist coupled branching random walks $Y_{t}^{k, A}$ and $Y_{t}^{k, B}$ with initial conditions $Y_{0}^{k, A}$ and $Y_{0}^{k, B}$ whose difference is bounded in absolute value by a branching random walk $Y_{t}^{k, C}$ with initial condition

$$
Y_{0}^{k, C}(x)=[\beta k] \mathbf{1}_{\sqrt{k} J} .
$$

Lemma 5. For any $\varepsilon>0$ and compact interval $J$ there exist $\beta, T>0$ such that if

$$
Y_{0}^{k} \leq \beta \mathbf{1}_{\sqrt{k} J} \quad \text { then } \quad P\left\{\operatorname{supsup}_{t \leq T} X_{x \in \mathbb{R}}^{k}(t, x) \geq \varepsilon\right\}<\varepsilon .
$$

Proof. Consider first a sequence of branching random walks with initial densities $X^{k}(0, \cdot)=2 \beta \mathbf{1}_{J^{*}}$, where $J^{*}$ is a compact interval containing $J$ in its interior. By Corollary 5 , for any $T>0$ the random functions $X^{k}(T+t, x)$ (with $t \geq 0$ ) converge to the density $X(T+t, x)$ of a standard Dawson-Watanabe process with initial density $X(0, x)=2 \beta \mathbf{1}_{J^{*}}(x)$. (Note: The variance parameter here is $\sigma^{2}=2 / 3$.) Hence, by Shiga's Lemma 4, if $T>0$ is sufficiently small then

$$
P\left\{\min _{x \in J} X^{k}(T, x) \leq \beta\right\}<\varepsilon \quad \text { and } P\left\{\sup _{t \in[T, 2 T]} \sup _{x \in \mathbb{R}} X^{k}(t, x) \geq 4 \beta\right\}<\varepsilon .
$$

It follows that for each $k$, the particle configuration at time $T$ is such that the renormalized density $X^{k}(T, \cdot)$ exceeds $\beta \mathbf{1}_{J}$, except on an event of probability $<\varepsilon$.

Now consider branching random walks with initial densities $\tilde{X}^{k}(0, \cdot)=\beta \mathbf{1}_{J}$. By monotonicity in initial configurations, the corresponding particle density processes $\tilde{X}^{k}(t, x)$ are dominated by the density processes $X^{k}(T+t, x)$ of the preceding paragraph, except on events of probability $<\varepsilon$. Consequently, by the second inequality in (80) and the Markov property,

$$
P\left\{\sup _{t \leq T} \sup _{x} \tilde{X}^{k}(t, x) \geq 4 \beta\right\}<2 \varepsilon .
$$

Proof of Theorem Q Assume now that the initial particle densities $f_{k}(\cdot):=X^{k}(0, \cdot)$ satisfy the hypotheses of Theorem 2 in particular, all have support contained in the compact interval $J$, and $f_{k} \rightarrow f$ uniformly for some continuous function $f$. By Corollary [5, for every $T>0$ the density processes $X^{k}(T+t, x)$ converge weakly to $X(T+t, x)$, where $X(t, x)$ is the density process of the 
Dawson-Watanabe process with initial density $f$. By Kesten's Theorem [16], $\varepsilon>0$ there is a compact interval $J^{*} \supset J$ such that for every $T>0$ and $k \in \mathbb{N}$, with probability at least $1-\varepsilon$, the function $X^{k}(T, \cdot)$ has support contained in $J^{*}$. By Shiga's Lemma, for any $\varepsilon>0$, if $T>0$ is sufficiently small,

$$
P\left\{\sup _{x}\left|X^{k}(T, x)-X^{k}(0, x)\right| \geq \varepsilon\right\}<\varepsilon .
$$

Hence, by Lemma 5 and a comparison argument, it follows that for any $\varepsilon>0$, if $T>0$ is sufficiently small,

$$
P\left\{\sup _{t \leq T} \sup _{x}\left|X^{k}(T+t, x)-X^{k}(t, x)\right| \geq \varepsilon\right\}<\varepsilon .
$$

Tightness of the sequence $\left\{X^{k}(t, x)\right\}_{t \geq 0}$ now follows from Corollary 5 .

3.4. Proof of Proposition 5: Preliminaries. The proofs of the estimates (70)-(71) are based on a simple recursive formula for the $m$ th moment of a linear functional $\left\langle Y_{n}, \psi\right\rangle:=\sum_{x} Y_{n}(x) \psi(x)$ of the particle density at time $n$. Observe that, for any integer $m \geq 1$, the $m$ th power $\left\langle Y_{n}, \psi\right\rangle^{m}$ is the sum of all possible products $\prod_{i=1}^{m} \psi\left(x_{i}\right)$, where $x_{i}$ is the location of a particle in the $n$th generation (particles may be repeated). For any such product, the $r \leq m$ particles involved will have a last common ancestor (LCE), situated at a site $z$ in the $k$ th generation, for some $k \leq n$. There are two possibilities: either there is just $r=1$ particle in the product, in which case it is its own LCE and $k=n$, or there are at least two particles, in which case the LCE belongs to a generation $k<n$. Conditioning on the generation $k$ and location $z$ of the LCE leads to the following formula (for bounded functions $\psi$ ):

$$
E^{x}\left\langle Y_{n}, \psi\right\rangle^{m}=\sum_{z} \mathbb{P}^{n}(x, z) \psi(z)^{m}+\sum_{k=0}^{n-1} \sum_{z} \mathbb{P}^{k}(x, z) \sum_{r=2}^{m} \kappa_{r} \sum_{\mathbf{m} \in \mathcal{P}_{r}(m)} F_{n-k}(\psi ; \mathbf{m} ; z)
$$

where

$$
\mathbb{P}(x, y)=\frac{1}{3} \mathbf{1}\{|x-y| \leq 1\}
$$

is the transition probability kernel for nearest neighbor random walk with holding, $\kappa_{r}$ is the $r$ th descending factorial moment of the offspring distribution (the expected number of ways to choose $r$ particles from the offspring of any particle), $\mathcal{P}_{r}(m)$ is the set of all integer partitions of $m$ with $r$ nonzero elements $m_{i}$, and

$$
F_{n}(\psi ; \mathbf{m} ; z):=3^{-r} \sum_{j_{i}=0,1,-1} \prod_{i=1}^{r} E^{z+j_{i}}\left\langle Y_{n}, \psi\right\rangle^{m_{i}} .
$$

Notice that in each term of the second sum in (81), $r \geq 2$, reflecting the fact that these terms correspond to final products with $r \geq 2$ distinct particles, in which the individual particles are repeated $m_{i}$ times. Since $\sum_{i=1}^{r} m_{i}=m$ and all $m_{i} \geq 1$, it follows that $m_{i}<m$ : this is what makes formula (81) recursive. The formula (83) accounts for the possibility that the $r$ offspring will jump to random sites adjacent to the location $z$ of the LCE. The appearance of powers $\mathbb{P}^{n}$ of the transition probability kernel of the nearest neighbor random walk with holding derives from the fact that the branching random walk is critical, so that the expected number of descendants at $(n, z)$ of an initial particle at $(0, x)$ is $\mathbb{P}^{n}(x, z)$. Following are standard estimates that will be used to bound such transition probabilities. 
Lemma 6. There exist constants $C, \beta<\infty$ such that for all $x, y \in \mathbb{Z}, n \in \mathbb{N}$, and $0 \leq \alpha \leq 1$,

$$
\begin{aligned}
\mathbb{P}^{n}(0, x) & \leq C n^{-1 / 2} \varphi_{n}(\beta x), \\
\left|\mathbb{P}^{n}(0, x)-\mathbb{P}^{n}(0, y)\right| & \leq C\left(n^{-1 / 2}|x-y| \wedge 1\right) \Phi_{n}(\beta x, \beta y), \\
\left|\mathbb{P}^{n}(0, x)-\mathbb{P}^{n+\alpha n}(0, x)\right| & \leq C n^{-1 / 2} \alpha \varphi_{n}(\beta x) .
\end{aligned}
$$

Proof. The first inequality follows from the local limit theorem and standard large deviations estimates for simple nearest-neighbor random walk. The second and third inequalities use also the fact that the Gauss kernel $\varphi(x)$ is uniformly Lipshitz in $x$.

Finally, we record some elementary inequalities for Gaussian densities:

Lemma 7. For any $\beta>0$ there exists $C<\infty$ such that for all $x \in \mathbb{Z}, n \geq 1$, and $k \geq n / 2$,

$$
\begin{gathered}
\sum_{y \in \mathbb{Z}} \varphi_{n}(\beta y) \leq C \sqrt{n} ; \\
\sum_{y \in \mathbb{Z}} \varphi_{k}(\beta y) \varphi_{n-k}(\beta x-\beta y) \leq C \sqrt{n-k} \varphi_{n}(\beta x / 4) ; \\
\exp \left\{-\beta x^{2} / 4 n\right\} \leq C_{\beta} \exp \left\{-\beta(x \pm 1)^{2} / 2 n\right\} .
\end{gathered}
$$

3.5. Proof of (70). The proofs of the inequalities in Proposition 5 will proceed by induction on the power $m$. In cases (70)-(71), the starting point will be formula (81); we will use the induction hypothesis to bound the factors in the products (83). In each case it will be necessary to analyze terms separately in the ranges $k \leq n / 2$ and $k>n / 2$. To prevent a proliferation of subscripts, we will adopt the convention that values of constants $C, \beta$ may change from one line to the next. In particular, in each inductive step we will relax the constant $\beta$ (from $\beta$ to $\beta / 2$ or $\beta / 4$ ) to account for differences \pm 1 in the arguments of exponentials: this is justified by (89) in Lemma 7 above.

For the proof of (70), use the function $\phi=\delta_{x}$ in formula (81). When $m=1$, the terms indexed by $0 \leq k<n$ in (81) all vanish, leaving $E^{0} Y_{n}(x)=\mathbb{P}^{n}(0, x)$. Thus, the inequality (70) follows for $m=1$ directly from Lemma 6. Assume now that (70) holds for all powers $<m$, where $m \geq 2$. By (81) and the induction hypothesis,

$$
E^{0} Y_{n}(x)^{m} \leq \mathbb{P}^{n}(0, x)+C \sum_{k=0}^{n-1} \sum_{z} \mathbb{P}^{k}(0, z) \sum_{\mathbf{m} \in \mathcal{P}(m)} \prod_{i=1}^{r}\left((n-k)^{-1+m_{i} / 2} \varphi_{n-k}(\beta(x-z))\right)
$$

where $\mathcal{P}(m)=\cup_{i=2}^{m} \mathcal{P}_{r}(m)$ is the set of all partitions of $m$ with at least two nonzero elements $m_{i}$. (Note that the constants $\kappa_{r}$ have been absorbed in $C$.) The initial term $\mathbb{P}^{n}(0, x)$ has already been disposed in the case $m=1$ (since the right side of the inequality (70) is nondecreasing in $m$, provided $C_{m} \uparrow$ and $\left.\beta_{m} \downarrow\right)$. Thus, we need only consider the terms $k<n$ of the second sum.

Consider first the terms $k \leq n / 2$. For indicies in this range, $n-k>n / 2$, and so the factors in the inner products can be handled by simply replacing each $n-k$ by $n$ (at the cost of a constant multiplier): Since the number $r$ of factors in each product is at least 2, and since the exponents $m_{i}$ in eaach interior product sum to $m$,

$$
\begin{aligned}
\sum_{k \leq n / 2} & \leq C n^{-2+m / 2} \sum_{k \leq n / 2} \sum_{z} \mathbb{P}^{k}(0, z) \varphi_{n}(\beta(x-z)) \\
& \leq C n^{-1+m / 2} \varphi_{n}(\beta x) .
\end{aligned}
$$

Now consider the terms $k>n / 2$. For such terms, $n-k$ is no longer comparable to $n$, and so the factors in the interior products cannot be estimated in the same manner as in the case $k \leq n / 2$. However, when $k>n / 2$ the transition probability $\mathbb{P}^{k}(0, z)$ can be estimated using the local limit 
theorem (84): hence, using the convolution inequality (88) of Lemma 7 and the induction hypothesis, and once again that each interior product has at least two factors,

$$
\begin{aligned}
\sum_{k>n / 2} & \leq C n^{-1 / 2} \sum_{k>n / 2} \sum_{z} \varphi_{k}(\beta z)(n-k)^{-2+m / 2} \varphi_{n-k}(\beta(x-z)) \\
& \leq C n^{-1 / 2} \varphi_{n}(\beta x) \sum_{k>n / 2}(n-k)^{-2+m / 2} \sqrt{n-k} \\
& \leq C n^{-1 / 2} \varphi_{n}(\beta x) n^{m / 2-1 / 2},
\end{aligned}
$$

as desired. This proves inequality (70).

3.6. Proof of (71). This is also by induction on $m$. For notational ease, set $\psi_{x y}=\delta_{x}-\delta_{y}$, where $\delta_{z}$ is the Kronecker delta, and set

$$
d_{n}(x, y)=(|x-y| / \sqrt{n}) \wedge 1 .
$$

Consider first the case $m=1$. By formula (81) and the local limit bound (85),

$$
\left|E^{0}\left\langle Y_{n}, \psi_{x y}\right\rangle\right|=\left|\mathbb{P}^{n}(0, x)-\mathbb{P}^{n}(0, y)\right| \leq C n^{-1 / 2} d_{n}(x, y) \Phi_{n}(\beta x, \beta y)
$$

for suitable constants $C, \beta$. Inequality (71) for $m=1$ follows easily.

Assume then that inequality (71) is valid for all positive integer exponents less than $m$. The first sum on RHS(81) (the terms with $k=n$ ) can be bounded above by $C n^{-1 / 2}\left(\varphi_{n}(\beta x)+\varphi_{n}(\beta y)\right)$ using Lemma 6, and this in turn is bounded above by RHS(71). Thus, we need only consider the second sum on RHS(81) (the terms $k<n$ ). In each of these terms, the interior products (83) have at least two factors, each with $m_{i} \geq 1$, and in each of these products the sum of the exponents $m_{i}$ is $m$. Consequently, by (81) and the induction hypothesis,

$$
\sum_{k=0}^{n-1} \leq C \sum_{k=0}^{n-1} \sum_{z} \mathbb{P}^{k}(0, z)|x-y|^{m / 5}(n-k)^{-2+m / 2-m / 10} \Phi_{n-k}(\beta x-\beta z, \beta y-\beta z) .
$$

Consider first the terms in the range $k \leq n / 2$ : for these terms, $n-k \geq n / 2$, and so $\Phi_{n-k}$ is comparable (after a relaxation of $\beta$ ) to $\Phi_{n}$. Hence,

$$
\begin{aligned}
\sum_{k=1}^{n / 2} & \leq C n^{-2} n^{m / 2-m / 10}|x-y|^{m / 5} \sum_{k=1}^{n / 2} \sum_{z} \mathbb{P}^{k}(0, z) \Phi_{n-k}(\beta x-\beta z, \beta y-\beta z) \\
& \leq C n^{-1} n^{m / 2-m / 10}|x-y|^{m / 5} \Phi_{n}(\beta x, \beta y),
\end{aligned}
$$

as desired. Now consider the range $k>n / 2$ : Here we use the local limit estimate for $\mathbb{P}^{k}(0, z)$ and the Gaussian convolution inequality (88) to obtain

$$
\begin{aligned}
\sum_{k=n / 2}^{n-1} & \leq C|x-y|^{m / 5} \sum_{k=n / 2}^{n-1} \sum_{z} \mathbb{P}^{k}(0, z) \Phi_{n-k}(\beta x-\beta z, \beta y-\beta z)(n-k)^{-2+m / 2-m / 10} \\
& \leq C n^{-1 / 2}|x-y|^{m / 5} \Phi_{n}(\beta x, \beta y) \sum_{k=n / 2}^{n-1}(n-k)^{-3 / 2+m / 2-m / 10} \\
& \leq C n^{-1} n^{m / 2-m / 10}|x-y|^{m / 5} \Phi_{n}(\beta x, \beta y) .
\end{aligned}
$$

The final inequality relies on the fact that the exponent $-1 / 2+m / 2-m / 10$ is positive for all $m \geq 2$. This proves (71). 
3.7. Proof of (72). The moment formula (81) can no longer be used, since the expectation on LHS(72) involves the state of the branching random walk at two different times. However, it is not difficult to derive an analogous formula: For any integer $m \geq 1$, the $m$ th power $\left(Y_{t}(x)-Y_{s}(x)\right)^{m}$ is a sum of products $\prod_{i=1}^{m} \psi\left(x_{i}\right)$, where each $x_{i}$ is the location of a particle in either the $t$ th or $s$ th generation, and $\psi\left(x_{i}\right)= \pm \delta_{x}\left(x_{i}\right)$, with the sign \pm depending on whether the particle is in the $t$ th or sth generation. As in formula (81), the particles involved in any such product must have a last common ancestor in some generation $k$ before the $s \wedge t$ th generation. Conditioning on the generation and location of this last common ancestor leads to the formula

$$
\begin{aligned}
E^{y}\left(Y_{s}(x)-Y_{t}(x)\right)^{m}= & \mathbb{P}^{s}(y, x)+(-1)^{m} \mathbb{P}^{t}(y, x) \\
& +\sum_{k=0}^{s \wedge t} \sum_{z} \mathbb{P}^{k}(y, z) \sum_{r=2}^{m} \kappa_{r} \sum_{\mathbf{m} \in \mathcal{P}_{r}(m)} G_{s-k, t-k}(x-z ; \mathbf{m})
\end{aligned}
$$

where $\kappa_{r}$ and $\mathcal{P}_{r}(m)$ have the same meanings as in the moment formula (811) and

$$
G_{s, t}(z ; \mathbf{m})=3^{-r} \sum_{j_{i}=0,1,-1} \prod_{i=1}^{r} E^{j_{i}}\left(Y_{s}(z)-Y_{t}(z)\right)^{m_{i}}
$$

We will use (91) to prove (72) by induction on the power $m$, using arguments similar to those used in proving (70) and (71). Consider first the case $m=1$ : in this case the last sum in (91) vanishes, leaving

$$
E\left(Y_{n}(x)-Y_{n+\alpha n}(x)\right)=\mathbb{P}^{n}(0, x)-\mathbb{P}^{n+\alpha n}(0, x) .
$$

Hence, inequality (72) follows immediately from estimate (86).

Assume now that (72) holds for all positive integer exponents smaller than $m$, for some integer $m \geq 2$. To prove that (72) holds for the exponent $m$, consider RHS(91), with $s=n$ and $t=n+\alpha n$. The sum of the first two terms coincides with (93). To verify that this sum, in absolute value, is smaller than RHS(72), observe that by (86) the sum is smaller than $C \alpha n^{-1 / 2} \varphi_{n}(\beta x)$; since $\alpha n$ must be an integer, $\alpha \geq n^{-1}$, and so

$$
n^{1 / 2} \alpha \leq n^{m / 2} \alpha^{m / 8}
$$

for all $m \geq 2$, as required by (72). Thus, it remains to prove that the sum of the terms with $r \geq 2$ in (91) is also bounded by RHS(72).

Note first that it suffices to consider values of $\alpha \leq 1 / 2$, because for $1 / 2 \leq \alpha \leq 1$ the factor $\alpha^{m / 5}$ on RHS(72) is bounded below. Now split the terms of the last sum in (91) into three ranges: first $k \leq n / 2$, then $n / 2 \leq k \leq n-n \alpha$, and finally $n-n \alpha \leq k<n$. In the range $k \leq n-n \alpha$, the induction hypothesis applies, because for these terms $\alpha^{\prime}=n \alpha /(n-k) \leq 1$. (Recall that one of the hypotheses of Proposition 5 is that $\alpha \leq 1$, so to use (72) in an induction argument the implied $\alpha^{\prime}$ cannot exceed 1$)$. For $k \leq n / 2$, the ratio $n /(n-k)$ is bounded above by 2 ; consequently,

$$
\begin{aligned}
\sum_{k \leq n / 2} & \leq C \sum_{k \leq n / 2} \sum_{z} \mathbb{P}^{k}(0, z) n^{-2+m / 2} \alpha^{m / 5} \varphi_{n-k}(\beta x-\beta z) \\
& \leq C n^{-2+m / 2} \alpha^{m / 5} \sum_{k \leq n / 2} \varphi_{n}(\beta x) \\
& \leq C n^{-1+m / 2} \alpha^{m / 5} \varphi_{n}(\beta x),
\end{aligned}
$$


which agrees with RHS(72). Next, consider terms in the range $n / 2 \leq k \leq n-n \alpha$ : By the induction hypothesis, the local limit bound (84), and the Gaussian convolution inequality (88),

$$
\begin{aligned}
\sum_{k=n / 2}^{n-n \alpha} & \leq C \sum_{k=n / 2}^{n-n \alpha} \sum_{z} \mathbb{P}^{k}(0, z)(n-k)^{-2+m / 2}(\alpha n /(n-k))^{m / 5} \varphi_{n-k}(\beta(x-z)) \\
& \leq C n^{-1 / 2} n^{m / 5} \alpha^{m / 5} \varphi_{n}(\beta x) \sum_{k=n / 2}^{n-n \alpha}(n-k)^{-3 / 2+m / 2-m / 5} \\
& \leq C n^{-1 / 2} n^{m / 5} \alpha^{m / 5} \varphi_{n}(\beta x) \sum_{j=1}^{n / 2} j^{-3 / 2+m / 2-m / 5} \\
& \leq C n^{-1+m / 2} \alpha^{m / 5} \varphi_{n}(\beta x) .
\end{aligned}
$$

The last inequality uses the fact that the exponent $-1 / 2+m / 2-m / 5$ is positive for all $m \geq 2$.

Finally, consider the terms in the range $n-n \alpha<k \leq n$. Here the induction hypothesis cannot be used, because $n \alpha / n-k>1$. Instead we use the bound (70), which we have already proved is valid for all $m$. This, together with (84) and (88), implies

$$
\begin{aligned}
\sum_{k=n-n \alpha}^{n-1} & \leq C \sum_{k=n-n \alpha}^{n-1} \sum_{z} \mathbb{P}^{k}(0, z)(n-k)^{-2+m / 2} \varphi_{n \alpha}(\beta x-\beta z) \\
& \leq C n^{-1 / 2} \varphi_{n}(\beta x) \sum_{k=n-n \alpha}^{n-1}(n-k)^{-3 / 2+m / 2} \\
& \leq C n^{-1+m / 2} \alpha^{-1 / 2+m / 2} \varphi_{n}(\beta x) \\
& \leq C n^{-1+m / 2} \alpha^{m / 5} \varphi_{n}(\beta x) .
\end{aligned}
$$

This completes the proof of (72).

Acknowledgments. The author thanks Regina Dolgoarshinnykh and Xinghua Zheng for useful discussions.

\section{REFERENCES}

[1] D. Aldous. Brownian excursions, critical random graphs and the multiplicative coalescent. Ann. Probab., 25(2):812-854, 1997

[2] K. B. Athreya and P. E. Ney. Branching processes. Springer-Verlag, New York, 1972. Die Grundlehren der mathematischen Wissenschaften, Band 196.

[3] L. Belhadji and N. Lanchier. Individual versus cluster recoveries within a spatially structured population. Ann. Appl. Probab., 16(1):403-422, 2006.

[4] P. Billingsley. Convergence of probability measures. Wiley Series in Probability and Statistics: Probability and Statistics. John Wiley \& Sons Inc., New York, second edition, 1999. A Wiley-Interscience Publication.

[5] J. T. Cox, R. Durrett, and E. A. Perkins. Rescaled voter models converge to super-Brownian motion. Ann. Probab., 28(1):185-234, 2000.

[6] D. A. Dawson. Geostochastic calculus. Canad. J. Statist., 6(2):143-168, 1978.

[7] D. A. Dawson and K. J. Hochberg. The carrying dimension of a stochastic measure diffusion. Ann. Probab., 7(4):693-703, 1979.

[8] D. A. Dawson and E. A. Perkins. Measure-valued processes and renormalization of branching particle systems. In Stochastic partial differential equations: six perspectives, volume 64 of Math. Surveys Monogr., pages 45-106. Amer. Math. Soc., Providence, RI, 1999.

[9] R. Dolgoarshinnykh and S. P. Lalley. Critical scaling for the sis stochastic epidemic. J. Applied Probabiliity, 43:892-898, 2006.

[10] R. Durrett. Stochastic spatial models. SIAM Rev., 41(4):677-718 (electronic), 1999. 
[11] R. Durrett, L. Mytnik, and E. Perkins. Competing super-Brownian motions as limits of interacting particle systems. Electron. J. Probab., 10:no. 35, 1147-1220 (electronic), 2005.

[12] R. Durrett and E. A. Perkins. Rescaled contact processes converge to super-Brownian motion in two or more dimensions. Probab. Theory Related Fields, 114(3):309-399, 1999.

[13] A. M. Etheridge. An introduction to superprocesses, volume 20 of University Lecture Series. American Mathematical Society, Providence, RI, 2000.

[14] S. N. Evans and E. A. Perkins. Explicit stochastic integral representations for historical functionals. Ann. Probab., 23(4):1772-1815, 1995.

[15] I. Karatzas and S. E. Shreve. Brownian motion and stochastic calculus, volume 113 of Graduate Texts in Mathematics. Springer-Verlag, New York, second edition, 1991.

[16] H. Kesten. Branching random walk with a critical branching part. J. Theoret. Probab., 8(4):921-962, 1995.

[17] N. Konno and T. Shiga. Stochastic partial differential equations for some measure-valued diffusions. Probab. Theory Related Fields, 79(2):201-225, 1988

[18] A. Martin-Löf. The final size of a nearly critical epidemic, and the first passage time of a Wiener process to a parabolic barrier. J. Appl. Probab., 35(3):671-682, 1998.

[19] D. Mollison. Spatial contact models for ecological and epidemic spread. J. Roy. Statist. Soc. Ser. B, 39(3):283$326,1977$.

[20] C. Müller and R. Tribe. Stochastic p.d.e.'s arising from the long range contact and long range voter processes. Probab. Theory Related Fields, 102(4):519-545, 1995.

[21] R. Schinazi. On the role of social clusters in the transmission of infectious diseases. Theor. Pop. Biol., 61:163-169, 2002.

[22] T. Shiga. Two contrasting properties of solutions for one-dimensional stochastic partial differential equations. Canad. J. Math., 46(2):415-437, 1994.

[23] J. B. Walsh. An introduction to stochastic partial differential equations. In École d'été de probabilités de SaintFlour, XIV-1984, volume 1180 of Lecture Notes in Math., pages 265-439. Springer, Berlin, 1986.

[24] S. Watanabe. A limit theorem of branching processes and continuous state branching processes. J. Math. Kyoto Univ., 8:141-167, 1968.

[25] E. T. Whittaker and G. N. Watson. A course of modern analysis. Cambridge Mathematical Library. Cambridge University Press, Cambridge, 1996. An introduction to the general theory of infinite processes and of analytic functions; with an account of the principal transcendental functions, Reprint of the fourth (1927) edition.

[26] X. Zheng. Spatial epidemics in higher dimensions. PhD thesis, University of Chicago, (in progress). 